\title{
Advanced Electro-Optic Surety Devices
}

Federal Manufacturing \& Technologies

C. E. Watterson

KCP-613-5369

Published May 1997

Topical Report

Approved for public release; distribution is unlimited.

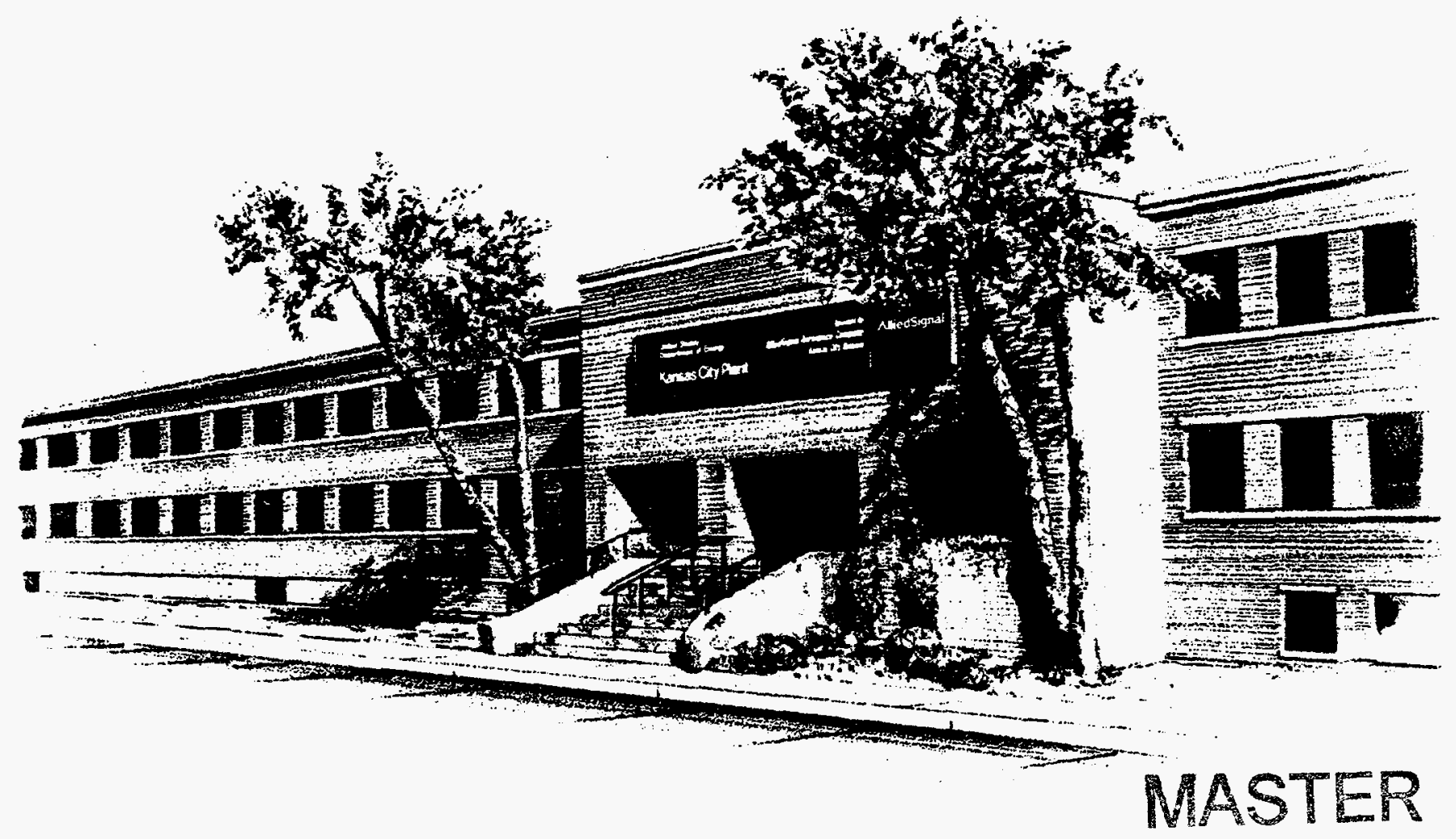

Prepared Under Contract Number DE-ACO4-76-DP00613 for the United States Department of Energy 


\section{DISCLAIMER}

This report was prepared as an account of work sponsored by an agency of the United States Government. Neither the United States Government nor any agency thereof, nor any of their employees, makes any warranty, express or implied, or assumes any legal liability or responsibility for the accuracy, completeness, or usefulness of any information, apparatus, product, or process disclosed, or represents that its use would not infringe privately owned rights. The views and opinions of authors expressed herein do not necessarily state or reflect those of the United States Government or any agency thereof.

All data prepared, analyzed and presented has been developed in a specific context of work and was prepared for internal evaluation and use pursuant to that work authorized under the referenced contract. Reference herein to any specific commercial product, process or service by trade name, trademark, manufacturer, or otherwise, does not necessarily constitute or imply its endorsement, recommendation, or favoring by the United States Government, any agency thereof or AlliedSignal Inc.

Printed in the United States of America.

This report has been reproduced from the best available copy.

Available to DOE and DOE contractors from the Office of Scientific and Technical Information, P. O. Box 62, Oak Ridge, Tennessee 37831; prices available from (615) 576-8401, FTS 626-8401.

Available to the public from the National Technical Information Service, U. S. Department of Commerce, 5285 Port Royal Rd., Springfield, Virginia 22161.

A prime contractor with the United States Department of Energy under Contract Number DE-ACO4-76-DP00613.
AlliedSignal Inc. Federal Manufacturing \& Technologies P. O. Box 419159 Kansas City, Missouri 64141-6159 


\title{
ADVANCED ELECTRO-OPTIC SURETY DEVICES
}

\author{
C. E. Watterson
}

Published May 1997

Topical Report

C. E. Watterson, Project Leader

Project Team:

C. T. Allen

R. A. Becker

D. E. Brown

P. E. Fry

A. W. Gordon

D. G. Laughlin

R. W. Kramer

P. E. Klingsporn

P. M. Mizik

D. N. Moore

M. W. Moore

E. V. Roos

B. Rosenblum

J. E. Smoot

J. H. Williams 


\section{DISCLAIMER}

Portions of this document may be illegible in electronic image products. Images are produced from the best available original docoment. 


\section{Contents}

Section

Page

Abstract

Summary

Discussion

Scope and Purpose $\ldots \ldots \ldots \ldots \ldots \ldots \ldots \ldots \ldots \ldots \ldots \ldots \ldots$

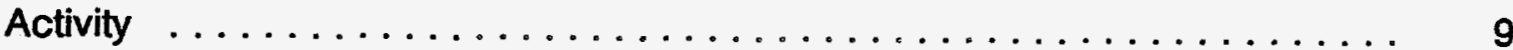

Package Design $\ldots \ldots \ldots \ldots \ldots \ldots \ldots \ldots \ldots \ldots \ldots \ldots$

External Lens-Fiber Array $\ldots \ldots \ldots \ldots \ldots \ldots \ldots \ldots \ldots \ldots \ldots$

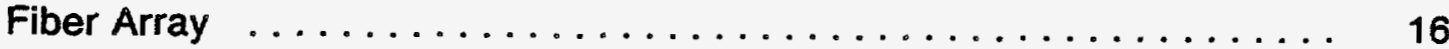

Laser-Drilled Fiber Array $\ldots \ldots \ldots \ldots \ldots \ldots \ldots \ldots \ldots \ldots \ldots$

Etched Quartz Two-Dimensional Array $\ldots \ldots \ldots \ldots \ldots \ldots \ldots \ldots$

Two-Dimensional V-Groove Array $\ldots \ldots \ldots \ldots \ldots \ldots \ldots \ldots \ldots \ldots$

Laser Modulator and Amplifier Array $\ldots \ldots \ldots \ldots \ldots \ldots \ldots \ldots$

Repackaged Laser Amplifier Array $\ldots \ldots \ldots \ldots \ldots \ldots \ldots \ldots \ldots$

Sandia Phase Modulator $\ldots \ldots \ldots \ldots \ldots \ldots \ldots \ldots \ldots \ldots$

Optical Switch Design $\ldots \ldots \ldots \ldots \ldots \ldots \ldots \ldots \ldots$

Accomplishments $\ldots \ldots \ldots \ldots \ldots \ldots \ldots \ldots \ldots \ldots \ldots \ldots \ldots \ldots \ldots \ldots$

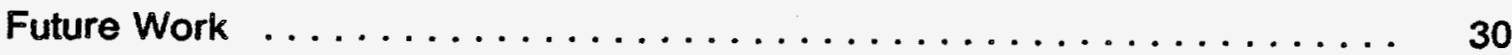

Reference $\ldots \ldots \ldots \ldots \ldots \ldots \ldots \ldots \ldots \ldots \ldots \ldots \ldots \ldots \ldots \ldots \ldots \ldots \ldots$

Appendix. Design Review of the Integrated Optical Maze $\ldots \ldots \ldots \ldots$ 



\section{Illustrations}

$\begin{array}{ll}\text { Figure } & \text { Page }\end{array}$

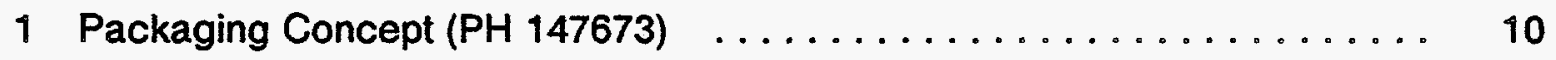

2 Gallium Arsenide Chip With Etched Mirror and Binary Lens . . . . . . 13

3 NSG $36 \times 36$ Microlens Array (PH 147973) $\ldots \ldots \ldots \ldots \ldots$

4 Divergence Measurement of NSG $36 \times 36$ Lens Array Aligned to $1 \times 12$ V-Groove Array of Single-Mode 28 Fiber . . . . . . . . . . 17

5 Single-Mode 28 Fibers in V-Groove Array (Magnified) $\ldots \ldots \ldots \ldots \ldots$

$6 \mathrm{CO}_{2}$ Laser Drilled Hole in 10 Mil Ceramic . . . . . . . . . . . . . 20

7 Excimer Laser Drilled Hole in 10 Mil Ceramic . . . . . . . . . . . . . 20

8 Etched Quartz Triads to Position Single-Mode Fibers . . . . . . . . . . 22

9 Single Triad on Etched Quartz Substrate . . . . . . . . . . . . . . 22

10 Ten-Channel Phase Modulator and Amplifier Array Chip (PH 147978) . . 23

11 Laser Amplifier Array Packaged on BeO Substrate (PH 147977) . . . . . 24

12 Repackaged Laser Amplifier Array $(\mathrm{PH} 148346) \ldots \ldots \ldots$

13 Laser Amplifier Array in Test Configuration (PH 148285) . . . . . . . . 27

14 Packaged Sandia Phase Modulator $(\mathrm{PH} 147678) \ldots \ldots \ldots$

$152 \times 2$ Lithium Niobate Optical Switch by AT\&T (PH 147675) . . . . . . . 29 


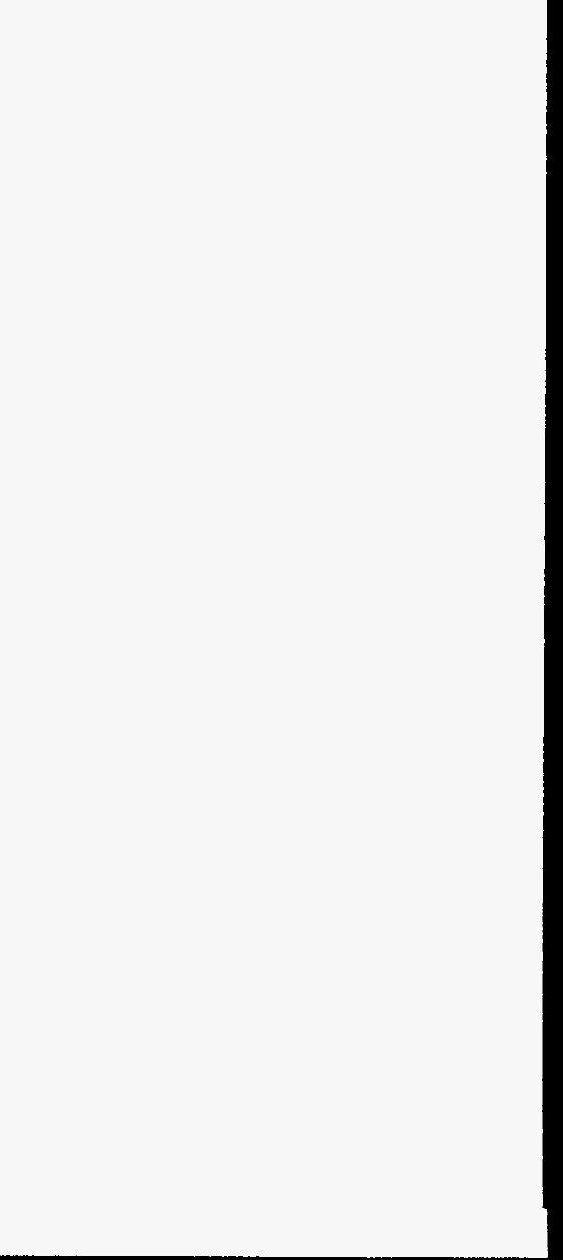




\section{Abstract}

The Advanced Electro-Optic Surety Devices project was initiated in March 1991 to support design laboratory guidance on electro-optic device packaging and evaluation. Sandia National Laboratory requested AlliedSignal Inc., Kansas City Division (KCD), to prepare for future packaging efforts in electro-optic integrated circuits. Los Alamos National Laboratory requested the evaluation of electro-optic waveguide devices for nuclear surety applications. New packaging techniques involving multiple fiber optic alignment and attachment, binary lens array development, silicon $V$-groove etching, and flip chip bonding were requested. Hermetic sealing of the electro-optic hybrid and submicron alignment of optical components present new challenges to be resolved. A 10-channel electro-optic modulator and laser amplifier were evaluated for potential surety applications.

\section{Summary}

The Advanced Electro-Optic Surety Devices project was initiated to develop packaging techniques to support development of Sandia electro-optic integrated circuits. The Sandia effort would include phase modulators, Mach-Zehnder interferometers, splitters, and couplers. To date, Sandia has provided phase modulators and requested that KCD assemble a fiber pigtailing station for assembly of these chips. Sandia is currently developing gallium arsenide devices with vertical-emitting collimated light. KCD has fabricated and delivered to Sandia a two-dimensional planar microlens and linear fiber optical array package.

In FY92, Los Alamos requested an expansion of the packaging effort to include the evaluation of appropriate electro-optic waveguide devices for nuclear surety applications. This has resulted in the evaluation of a 10-channel modulator and laser amplifier array, developed by McDonnell Douglas Electronic Systems Division for Phillips Laboratories.
Packaging efforts include die attach and wire bonding of gallium arsenide laser diodes, Mach-Zehnder interferometers, phase modulators, and a 10-channel laser amplifier modulator array. A six-axis, 40 nanometer resolution micropositioner station has been assembled and used to actively align polarization-maintaining fiber to gallium arsenide laser diodes within a fraction of 1 micron.

KCD has developed the capability to gold plate single-mode fiber optics. In addition, gold-plated ceramic ferrules have been acquired to allow soldering single-mode fibers through the wall of hybrid microcircuits. This capability can be used in-house for characterization of electrooptic devices.

A planar lens array to accept collimated light from an electro-optic integrated circuit and re-image into a single-mode fiber linear array has been procured. This lens array will complement Sandia's current efforts to etch a binary lens onto the waveguide substrate. The combination of 
Sandia's binary collimating lens and KCD's hermetic packaging approach, with the planar lens array, will provide a high tech state-of-the-art solution to this industry-wide problem.

A linear fiber optic array using Corning single-mode fibers and silicon $V$-grooves has been fabricated. A gold-silicon eutectic bond process has been tested for fabricating a high temperature glass microlens array with linear single-mode fiber optic array output. A $36 \times 36$

microlens array was aligned and packaged with a 15 single-mode fiber linear array and was delivered to Sandia for analysis.

A 10-channel electro-optic modulator and laser amplifier array, the U. S. Air Force (USAF) Phillips Laboratory PILOT chip, was procured from McDonnell Douglas and USAF Phillips Laboratory for evaluation. The necessary facilities to evaluate this device have been assembled. Initial testing of the pilot chip has been completed. Potential applications have indicated possible modifications to this device that will be proposed for future funding. Packaging requirements have been identified and started for two gallium arsenide die.

Fiber optic pigtailing is being evaluated to assemble electro-optic integrated circuits for device characterization. A microscope alignment and pigtailing station, modeled after one at Sandia, has been specified and is currently being purchased for future activities on this project. This same alignment station will be used to align the planar lens array to the electro-optic hybrid.

Etched V-groove silicon wafers have been evaluated for alignment of multiple singlemode fibers to the planar lens array. A second alignment system, using precision laser-machined holes in 10 mil ceramic, is being evaluated for multimode fiber alignment. Initial efforts with a $\mathrm{CO}_{2}$ laser provided sufficient accuracy to align multiple multimode fiber optics to a planar lens array. However, single-mode fiber will require higher precision of both hole formation and location. An excimer laser was evaluated for this purpose. 


\section{Discussion}

\section{Scope and Purpose}

The Advanced Electro-Optic Surety Devices project was initiated to develop processes required to package electrooptic integrated circuits from Sandia and to evaluate electro-optic waveguide devices for nuclear surety applications.

Several technologies, including polarization fiber optics, flip chip bonding, silicon V-groove etching, planar lens array fabrication and alignment, gold plating of fiber optics, submicron alignment of optical components, and laser ablation of precision holes in ceramic were all new to $\mathrm{KCD}$ at the start of this project. All these processes will be developed or purchased to support the Sandia compound semiconductor production program.

Project objectives are expected to expand as different electro-optic integrated circuits are created. The primary considerations are to provide a hermetically sealed hybrid integrated circuit with single or multiple fiber input/output (1/O). Optical system alignment should be as automated as possible, with computer-controlled active alignment when required. Future efforts also include device characterization to enhance engineering understanding of the devices being packaged.

Current activities include device characterization of the pilot chip, a 10 channel phase modulator/laser amplifier manufactured for the Air Force Phillips Laboratories by McDonnell Douglas Electronic Systems division. Optical and electronic parameterization of this device is interesting because of its potential use in nuclear surety. It is a state-of-the-art electro-optic integrated circuit which will exercise KCD's ability to package these devices. A custom thin film network is currently being fabricated to package the chip. Die attach, wire bond, fiber alignment, fiber array alignment, and device characterization are several of the manufacturing disciplines that will be exercised.

\section{Activity}

\section{Package Design}

The design of RF hybrid microelectronic packaging is based on hermeticity, electrical pin out, thermal, and microwave requirements. Photonic integrated circuits require that additional fiber optic and imaging lens systems be included in the design. The micron features of the optical waveguides, used in photonics, require precision alignment to input/output laser signals. Fiber optics, used in photonic integrated circuits and coherent communication systems, are often of the polarization-maintaining variety, with core alignment requirements of 0.1 micron or less.

Historically, hermetic photonic packages used metallized fiber optics passing through the package wall. These metallized fibers were soldered to the assembly for sealing. The planar lens approach would eliminate fiber optics entering the hermetic environment from outside the package. Embrittlement of the glass fibers during packaging, flaking of the gold metallization off the fiber surface, and general fragility of polarization fibers led to a search for a better packaging scheme for photonic integrated circuits. The 
general approach suggested as a viable alternative is shown in Figure 1.

A standard flatpack microelectronic package with microwave stripline pin 1/O houses the gallium arsenide photonic circuit. A transparent sapphire lid solders to the microelectronic package to provide the hermetic seal as well as a medium to transfer the laser signals in and out of the package. The gallium arsenide photonic circuit is flip chip bonded to the underside of the transparent sapphire lid.

The transparent sapphire lid is fabricated with a gold metallization layer around the rim. Gold pads for flip chip bonding of the gallium arsenide circuitry and additional metallization for circuit connections to the chip and the $1 / O$ pins are fabricated on the sapphire substrate. In the production environment, the completed metallized sapphire lid would be gold-tin soldered to

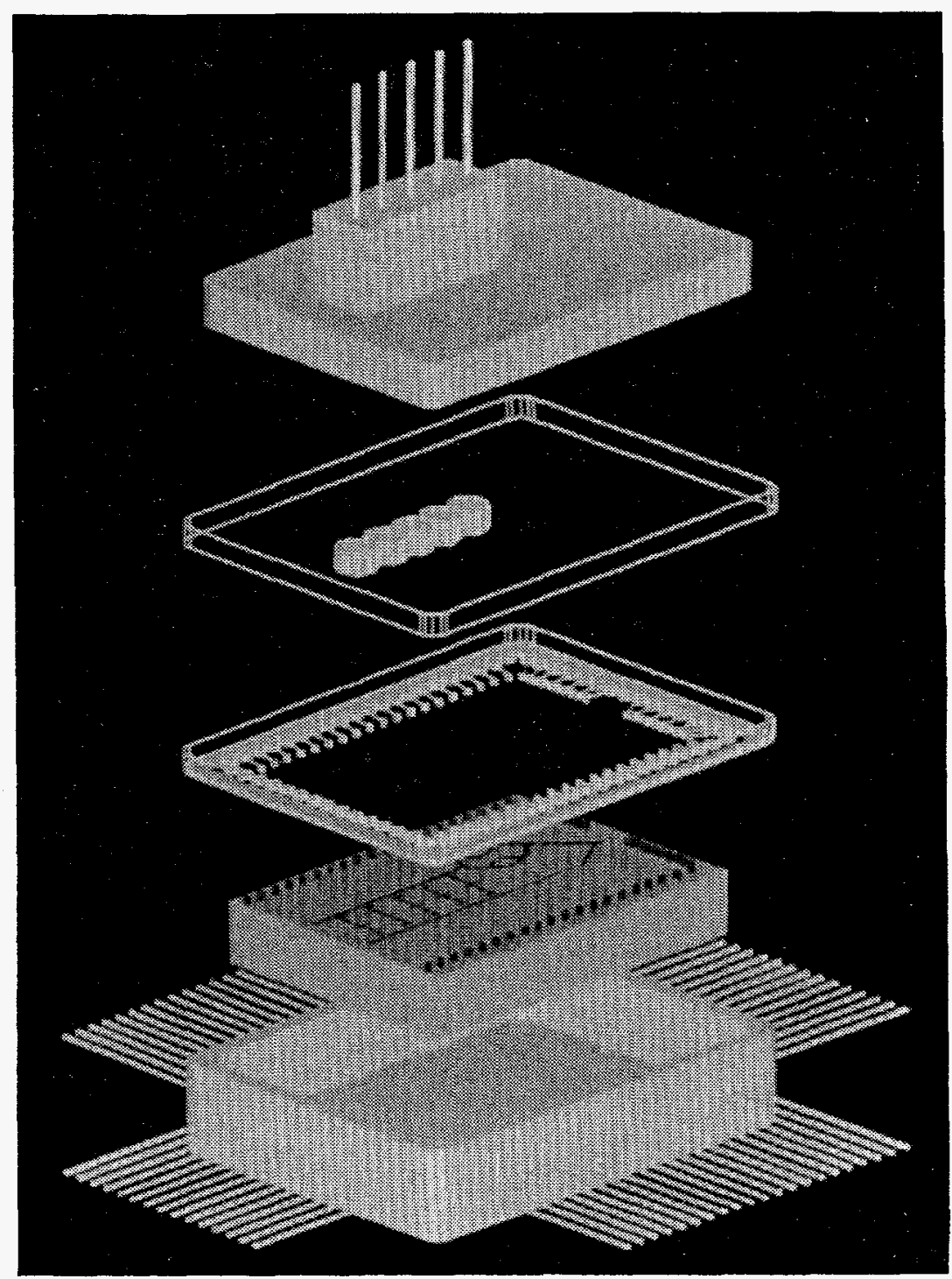

Figure 1. Packaging Concept 
the top of the flatpack in a nitrogen atmosphere with temperature and pressure applied. The flatpack would be purchased with a gold-tin solder preform tack welded to the top of the flatpack for prealignment. The flatpack would be assembled without a bottom plate.

Flip chip bonding of the gallium arsenide chip to the flatpack-sapphire lid assembly would provide some degree of automatic chip alignment. However, with the packaging concept (Figure 1), precision alignment of the gallium arsenide chip is not required at this stage of production. After insertion of the chip, ribbon or wire bonding between metallization pads on the sapphire substrate and the $1 / O$ pins would be completed. Finally, the flatpack bottom lid would be soldered in the same pressure temperature press as the sapphire lid. At this stage, a complete hermetic package is available for external assembly of a planar lens array.

The first planar lens array contains a single lens for accepting collimated laser input. The additional lens, on the first array, provides the multiple semi-collimated outputs from the chip. Precision fabrication of this first lens array is a requirement. Lithographic precision can be applied to the gallium arsenide chip.

The same precision is required of this first lens array, regardless of its optical configuration. This precision is possible with both conventional and state-of-the-art lens arrays. Specific lens systems will be discussed later in this analysis. A spacer may be required between the sapphire lid and the first planar lens array. With adequate precision in chip and planar lens fabrication, the optical alignment can be achieved by aligning the single laser input lens and one of the output lenses. The planar lens array and spacer could be bonded in place with epoxy. At this stage, a complete electrical optical test of the chip is possible.
The uppermost transparent plate (Figure 1) has a fiber optic block attached. This plate, with fiber optics, could be prealigned to the second planar lens array on the optical bench. After the second planar lens array and the transparent plate/fiber block were bonded, the entire top assembly would be actively aligned to the flatpack assembly.

The resulting photonic package would meet mil specification requirements and provide a compact assembly. Minor variations on this packaging concept are presented in this document to facilitate different lens approaches.

Certain assumptions concerning gallium arsenide chip design were incorporated in this packaging concept. An etched mirror must be incorporated in the chip manufacture to reflect waveguide input and output perpendicular to the plane of the chip (see Figure 2). A critical assumption is that this etched mirror could be manufactured with the angular precision required to interface a lens array to the chip. Etched mirror designs in gallium arsenide die have been successively fabricated by TRW Inc., ${ }^{1}$ showing the validity of this approach. Sandia is currently fabricating these chips.

\section{External Lens-Fiber Array}

The collimated beams entering and exiting the hermetic package depicted in Figure 2 require an external lens array and fiber array to input and output optical data. The lens array chosen is a $36 \times 36$ array of microlenses produced by NSG (see Figure 3a). Each lenslet is 250 micron diameter on 250 micron centers. The numerical aperture of each lenslet is 0.21 . A linear fiber array was constructed consisting of 15 single-mode fibers sandwiched between two silicon plates, with precision V-grooves for positioning the fibers. This lens matrix and fiber array were aligned and bonded 



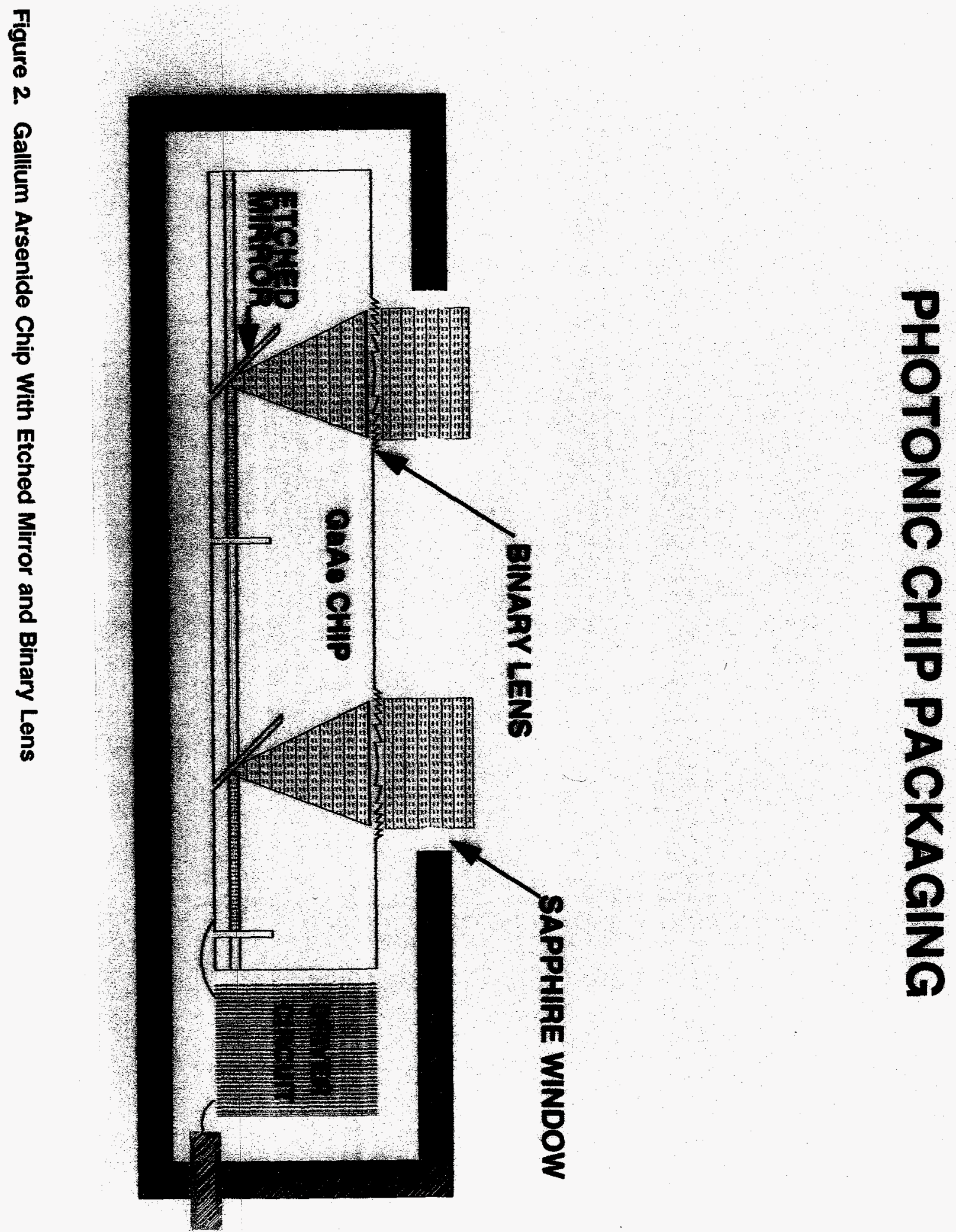


. 


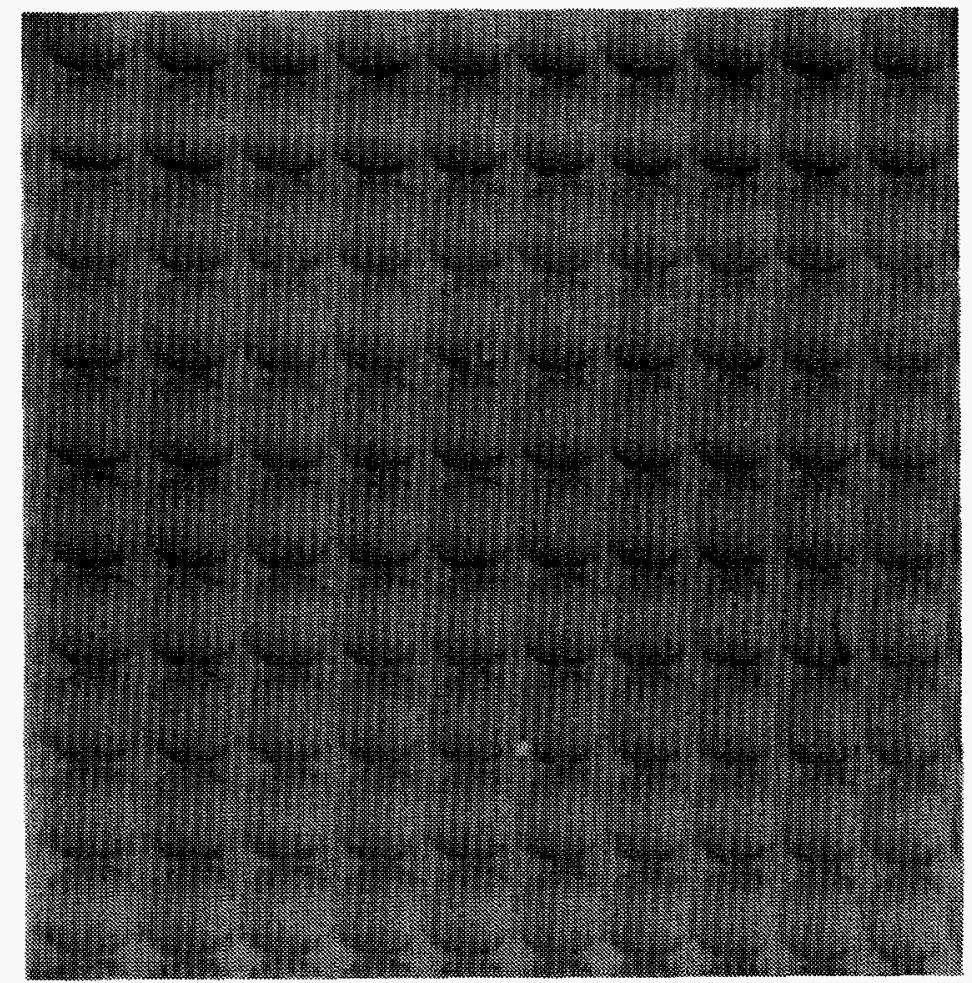

a. Lens Matrix

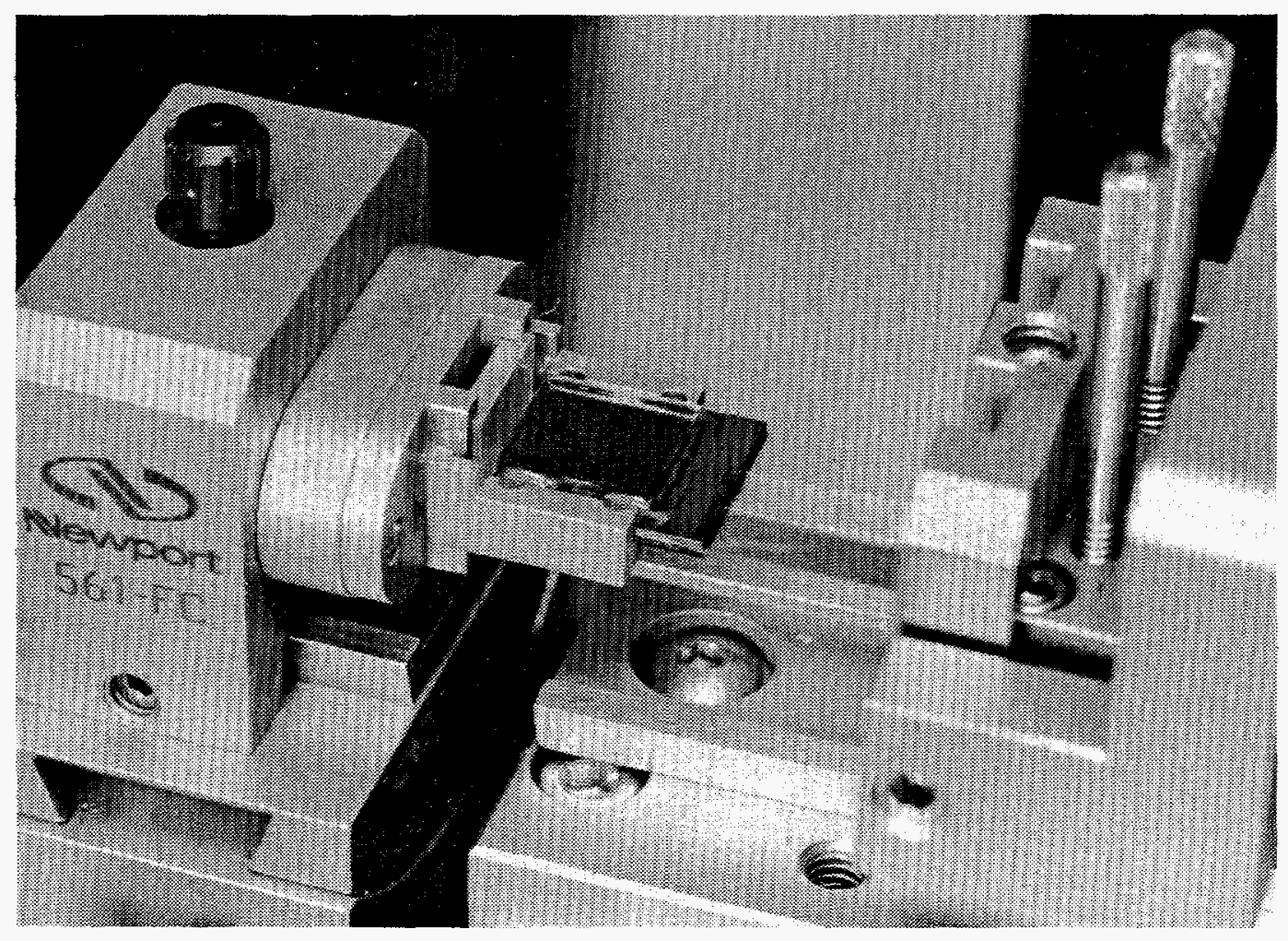

b. Lens Matrix and Fiber Array in Alignment Fixture

Figure 3. NSG $36 \times 36$ Microlens Array 
together to form a single assembly, shown in Figure $3 b$.

Each lenslet in the NSG array has a numerical aperture of 0.21 , which is not a perfect match for the Corning single-mode 28 fiber (NA 0.13). However, this is the only lens available at this time. A concurrent binary lens development at Sandia should provide binary lens arrays for future products, as required. The proton exchange lens arrays from NSG are available in a number of different numerical apertures, as a custom order. A full angle divergence of $\mathbf{4 0}$ milliradians was measured for a single lenslet in the NSG array. This data, shown in Figure 4, was obtained by illuminating a single fiber in the linear array and measuring full angle divergence of a single lenslet with Beamcode $^{\mathrm{m}}$ (Big Sky Software).

The relatively large divergence of the lenslets in this array reduce the overall efficiency of the system but reduce the alignment sensitivity concurrently. This trade off in design parameters can be applied to future lens array design.

The alignment fixturing, partly seen in Figure 3, was custom designed and fabricated at KCD around commercial three-axis stages from Newport Corporation. The custom fixture is capable of six degree of freedom alignment of the fiber array to the lens array. The custom fixturing rigidly maintains the distance of the lens array to the fiber array. This is important because adhesive can misalign the system during the curing process. Submicron positioning of the fiber array is possible using this fixturing.

Optical throughput was measured for each of the 15 fibers during the curing process. Only one fiber showed a variation in excess of 10 percent from start to finish. The other 14 were considerably better. This result is not surprising, in light of the high precision with which the linear fiber array was fabricated.

Successful fabrication of this device proves the feasibility of the hermetic package design concept shown in Figures 1 and 2. Possible variations on the gallium arsenide chip design of Figure 2, such as a separate internal binary lens array or the use of a more conventional lens array inside the package, make this a viable solution to an industry-wide problem.

\section{Fiber Array}

The linear single-mode fiber optic array was fabricated at KCD using chemically etched $\mathrm{V}$-grooves and Corning single-mode fiber. The Corning single-mode fiber was selected by Corning to have less than 1 micron diameter and concentricity variation. The chemically etched silicon V-grooves were fabricated by Wave Optics, Inc. Stock V-grooves are available with up to 32 grooves. The fibers tend to self align when placed in the $V$-grooves to provide a precision linear array. A magnified photo of a V-groove array is shown in Figure 5.

The fiber array shown in Figure 3 was bonded together with epoxy and end polished. The center-to-center spacing of the fibers was $250 \pm 0.68$ microns. The mode size of the single-mode 28 fiber, at 1320 nanometers, is 8.3 microns. This is sufficient precision to align linear arrays of single-mode fibers, laser diode arrays, waveguide arrays, and other optics requiring submicron positioning.

The V-groove array shown in Figure 5 was assembled using a silicon-gold eutectic bond. The fibers were chrome/gold plated. The $V$-grooves were plasma etched and gold plated. The $V$-grooves and fibers were assembled into an array and heated until the silicon-gold eutectic formed. After end polishing, a high temperature linear array results with this technique. Initial 


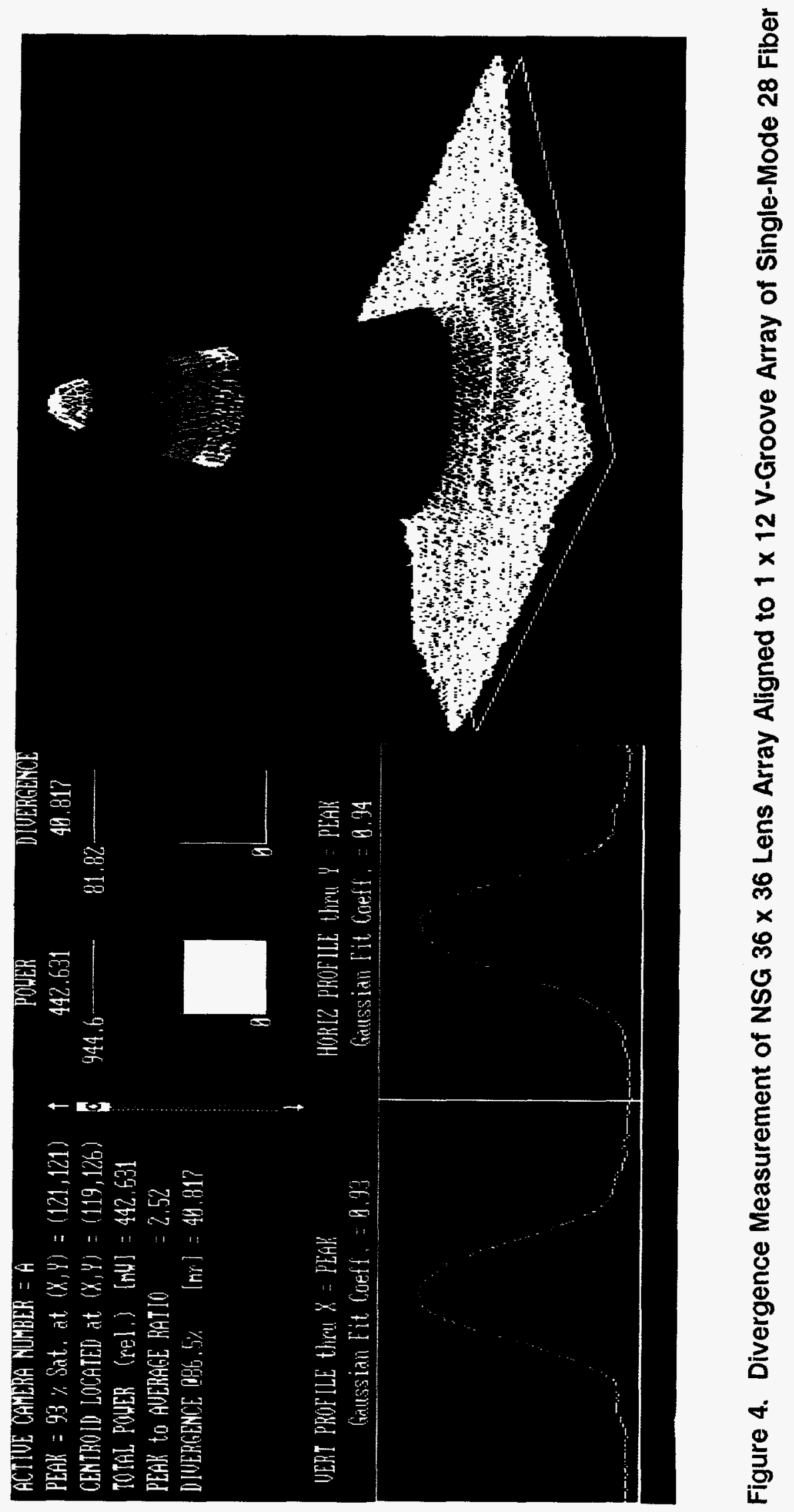




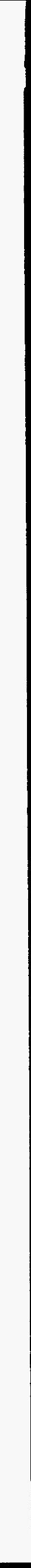




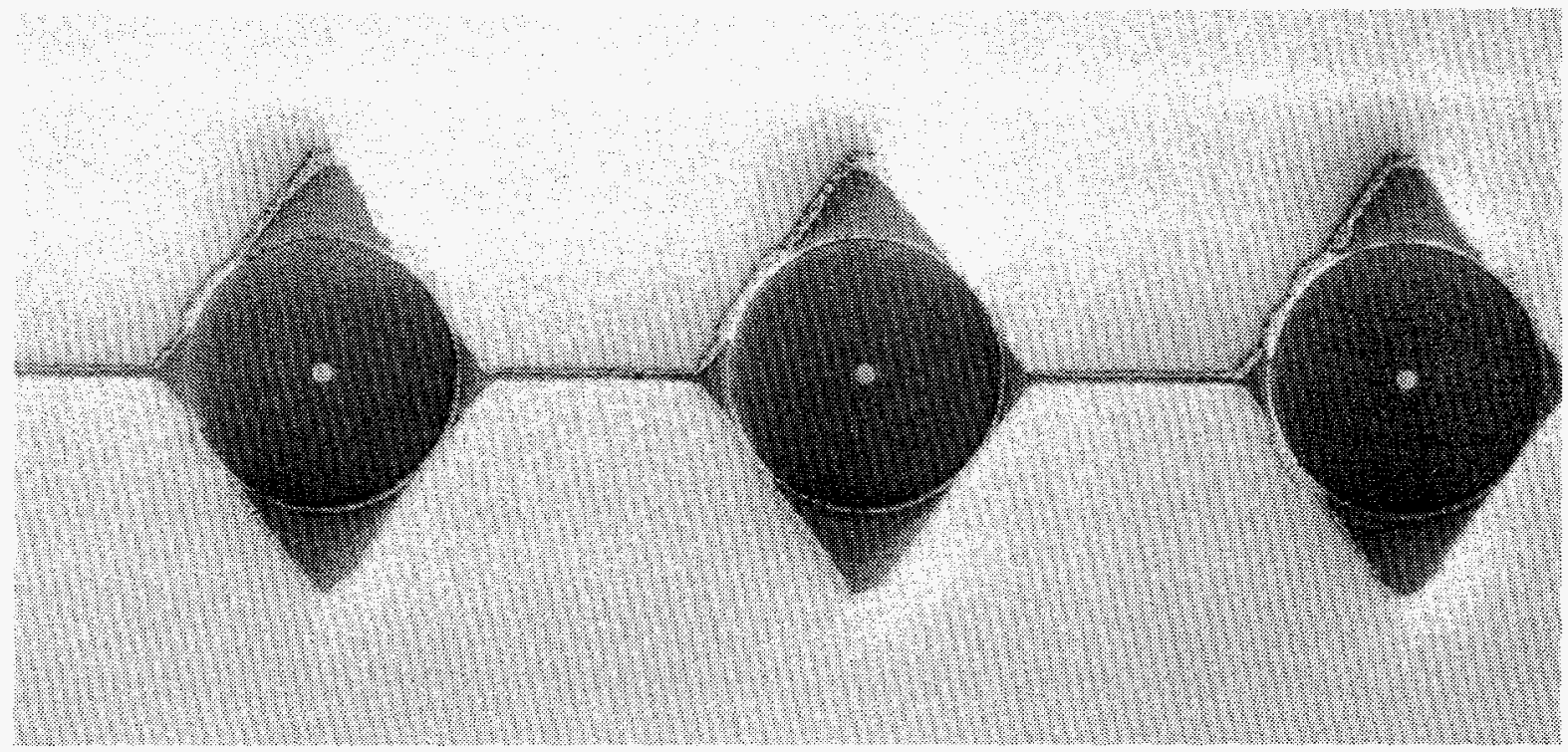

Figure 5. Single-Mode 28 Fibers in V-Groove Array (Magnified)

center-to-center spacings were not as precise as those obtained from the epoxybonded assembly. A continuing effort is planned to improve the spacing. Additional efforts are planned using polarization fiber obtained from $3 \mathrm{M}$, with diameter and concentricity tolerances similar to the Corning fiber. Most electro-optic integrated circuits require polarizing fiber at the optical input.

\section{Laser-Drilled Fiber Array}

Arrays of 126 and 127 micron holes were drilled in ceramic substrates to evaluate this as a technique for packaging fibers in a linear array. Both $\mathrm{CO}_{2}$ and excimer lasers were employed. Stripped fibers were inserted into the drilled holes and examined visually. Figure 6 shows a fiber inserted into a $\mathrm{CO}_{2}$ drilled hole. Figure 7 shows a fiber inserted into an excimer drilled hole.

The excimer drilled holes were much cleaner than the $\mathrm{CO}_{2}$ drilled holes, but the geometrical location of the fiber optic was inadequate in both cases for the alignment of the $\mathbf{8 . 3}$ micron central core of a singlemode fiber. Similar results were obtained at Sandia for holes drilled in crystalline silicon. All three sets of holes would be adequate for locating multimode fibers.

Both the $\mathrm{CO}_{2}$ and the excimer laser drilling facilities at KCD are programmable and can drill holes in a user-definable array. This capability may be used in future efforts to solder fibers with active alignment systems.

Two-dimensional single-mode fiber arrays are required for optical computers, optical correlation systems for pattern recognition, vertical cavity-emitting laser applications, and others. Extensive manufacturing of vertical cavity-emitting laser arrays, at Sandia, have resulted in a request to KCD to develop the two-dimensional fiber arrays. The laser drilling approach does not appear to hold much promise for single-mode fiber arrays. Precision chemical etching of quartz substrates is the next approach that will be tested. Stacking of dual-sided V-groove arrays is also being considered for constructing twodimensional fiber arrays. 


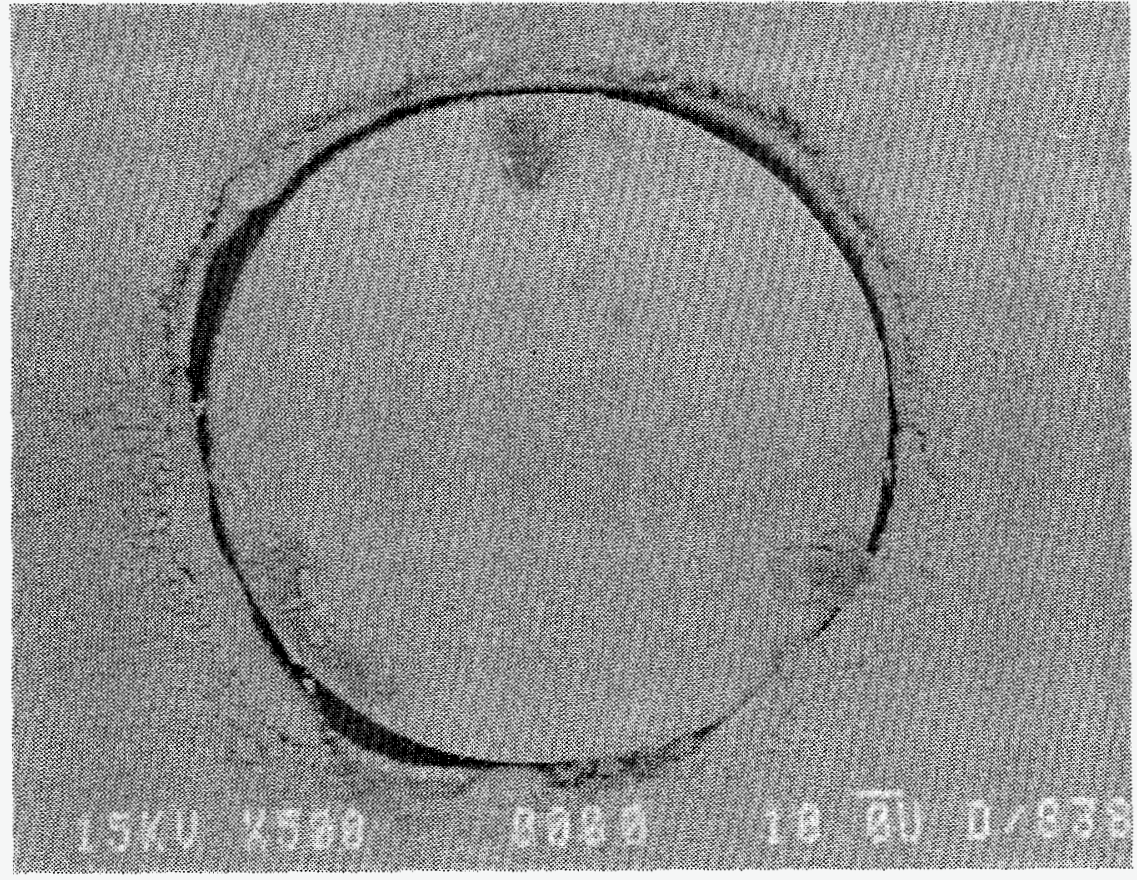

Figure 6. $\mathrm{CO}_{2}$ Laser Drilled Hole in 10 Mil Ceramic

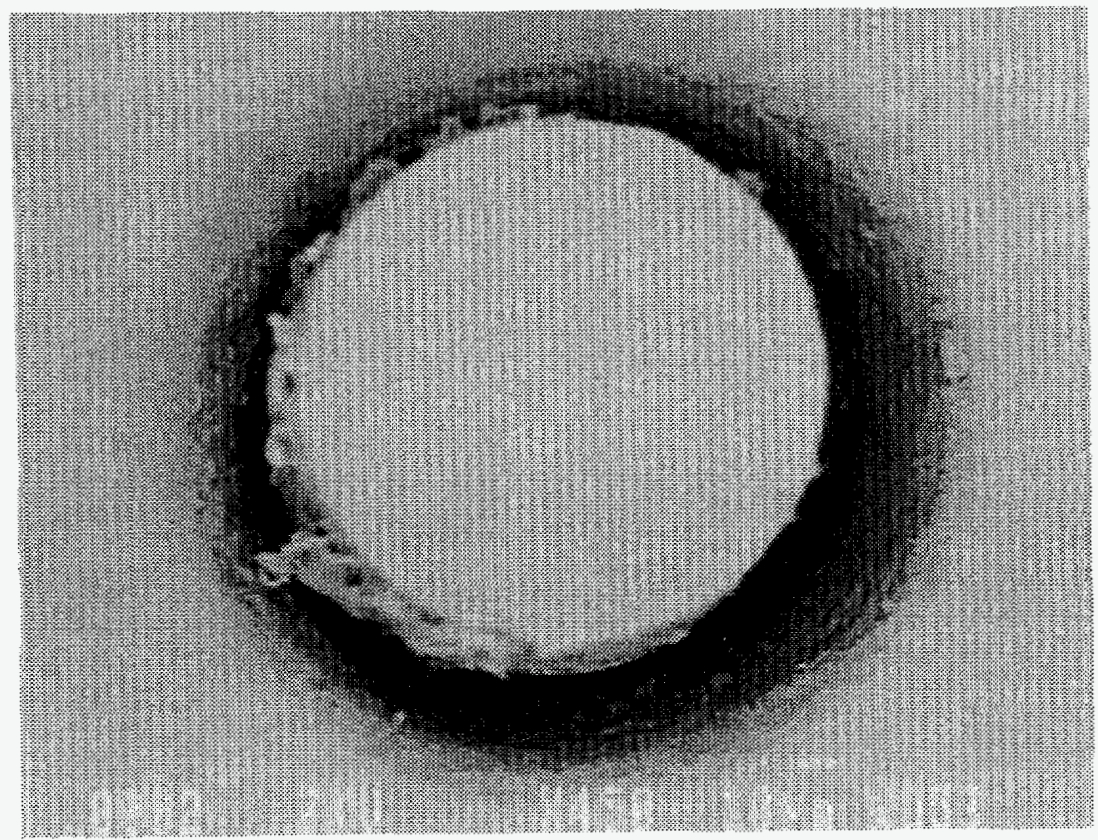

Figure 7. Excimer Laser Drilled Hole in 10 Mil Ceramic 
Etched Quartz Two-Dimensional Array

An etched quartz array was fabricated in accordance with KCD design by Photonic Integration Research Inc. The array, shown in Figure 8, consists of triad pillar elements on $\mathbf{5 0 0}$ micron centers designed to position individual fibers. Each triad of quartz pillars is designed to precisely position 125 micron fiber. The transcribed cylinder, which just fits inside the three pillars, was designed to be 126,127 , and 128 microns. The individual fibers would be located using a microscope and fixed in position with UV cure epoxy. The actual assembly of this fiber array will be performed in FY94.

A close up view of a single triad is shown in Figure 9.

\section{Two-Dimensional V-Groove Array}

Sandia has fabricated silicon V-grooves, similar to those used in the fiber array assembly seen in Figure 5. The Sandia silicon wafers, however, have V-grooves on both sides. Their silicon fabrication foundry has the capability to precisely register photomasks on opposite sides of a substrate. By inserting precision singlemode fibers into the $V$-grooves and stacking silicon wafers, a two-dimensional precision array is possible. Several of these wafers have been delivered to KCD for assembly of these arrays. Precision measurements of the original V-groove wafers and the completed cable assemblies have been requested.

\section{Laser Modulator and Amplifier Array}

A 10-channel phase modulator and amplifier array chip (Figure 10), manufactured by McDonnell Douglas Electronic Systems Division for the USAF Phillips Laboratory Semiconductor Laser Applications Branch, was chosen for packaging and optical/electrical characterization. This gallium arsenide chip represents the state of the art in electro-optic device fabrication. It employs waveguide technology capabilities encompassing saturable absorption and laser amplification, phase modulation, intensity modulation, and 1-to-10 splitting. $Y$-branch amplification is employed in the splitter to uniformly propagate the optical energy throughout the 10-channel distribution network. Reactively ion etched turning mirrors are employed to achieve 90 degree beam deflection.

The chip shown in Figure 10 was designed for a wavelength of $\mathbf{8 5 0}$ nanometers. It is a coherent device capable of controlling the phase or intensity of each individual channel. The 10 final amplifiers are ganged together as a result of the common metallization but could be individually controlled in an alternate design. Singlemode polarizing fiber is required for the optical input, upper right hand corner. The device currently being evaluated has a threshold input of 8 milliwatts and a maximum input of $\mathbf{2 0}$ milliwatts, as measured directly out of the input fiber. Several decibels of coupling loss are assumed at the input. The maximum output of each channel, which varies from device to device, is in the range of 40 to 75 milliwatts per channel. The phase modulators can also be used as intensity modulators and are individually addressable. The laser diode used to drive this chip was a Spectra Diode Labs SDL 5422-H1.

Two stages of isolation were required to maintain the laser diode-amplifier array in single-frequency operation. The singlefrequency operation obtained was stable, however. 3M PZ polarizing fiber was used on the input.

The original packaging of the amplifier array is shown in Figure 11. A beryllium oxide substrate and gold wire bonding 


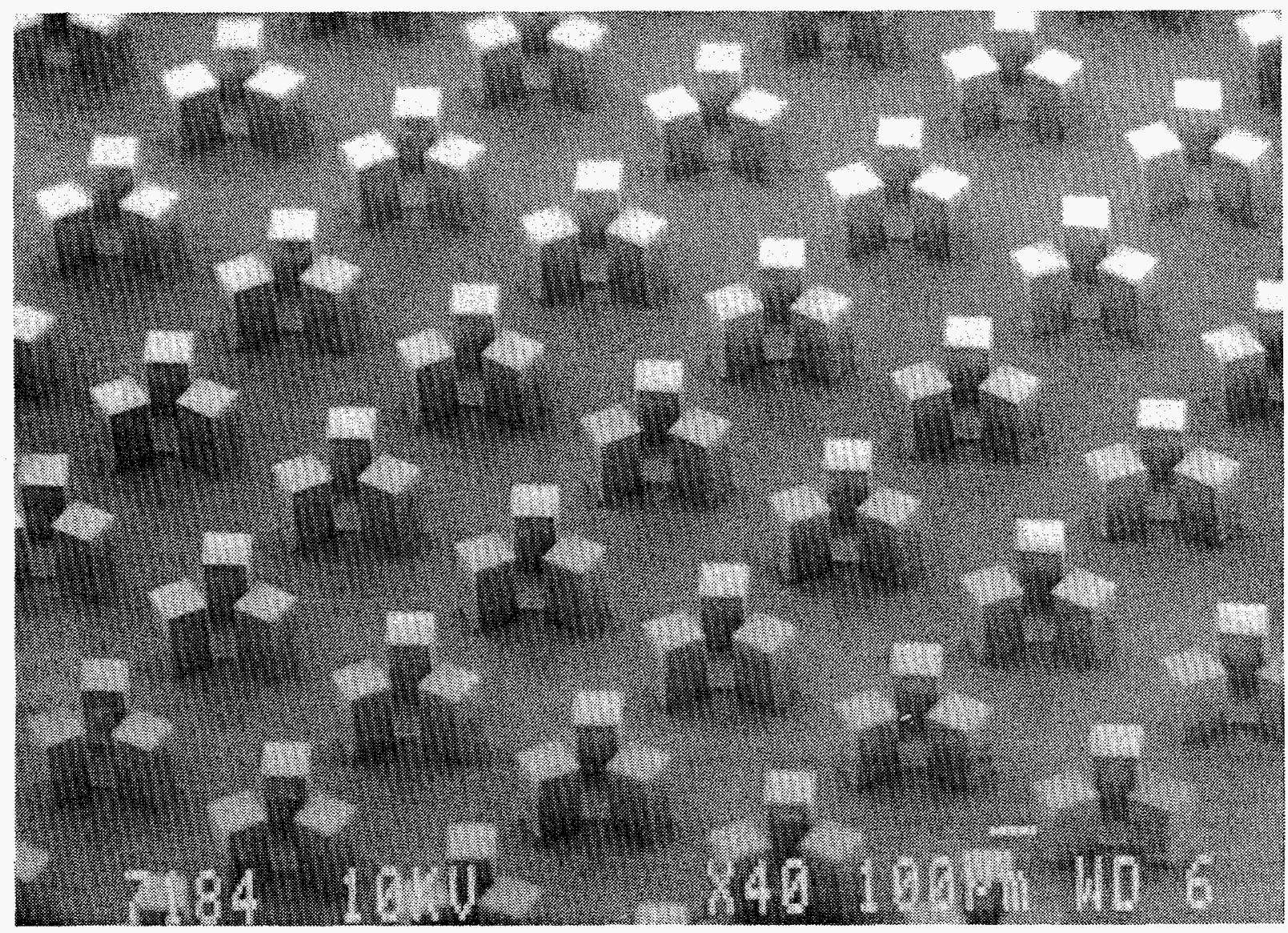

Figure 8. Etched Quartz Triads to Position Single-Mode Fibers

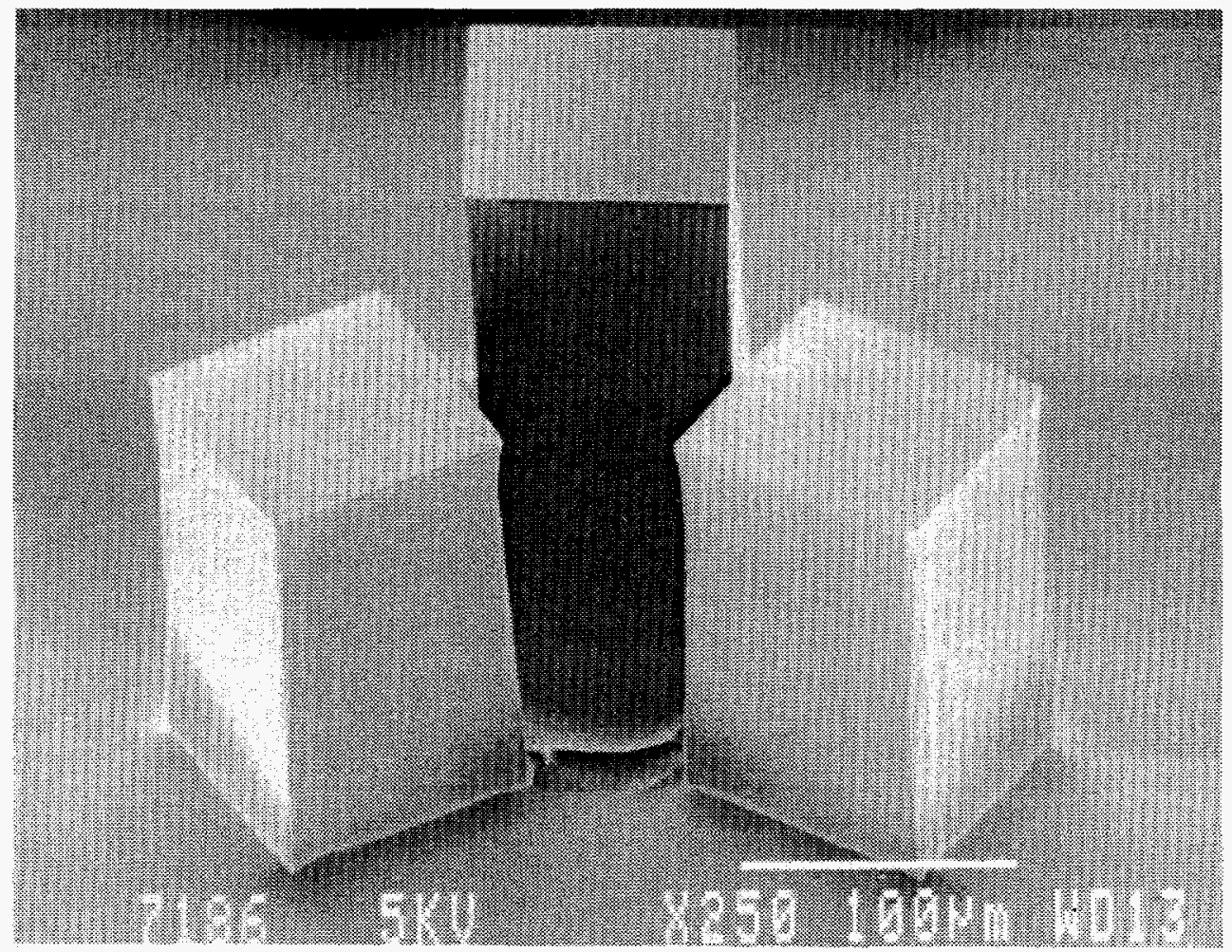

Figure 9. Single Triad on Etched Quartz Substrate 


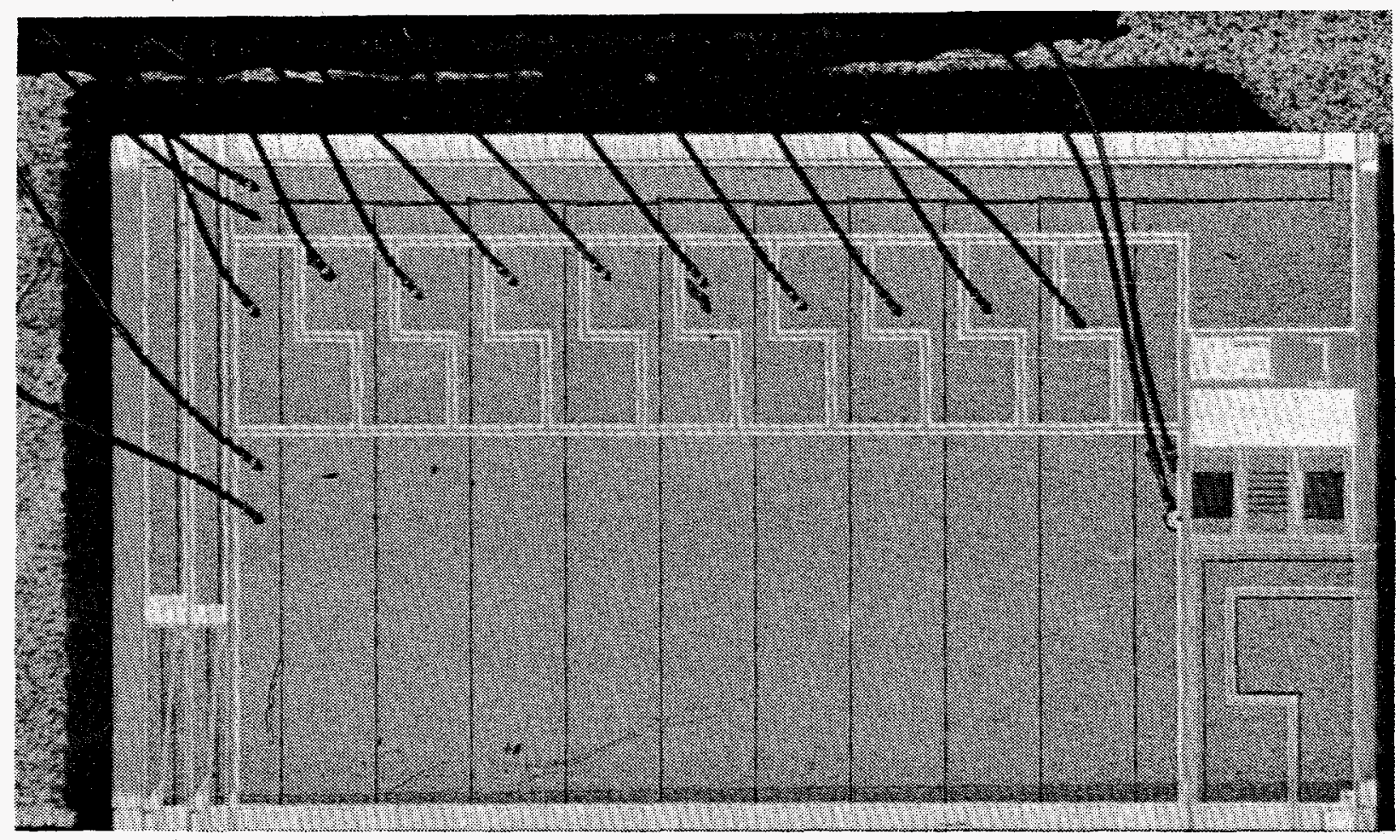

a. Laser Splitter, Modulator, and Amplifier Array

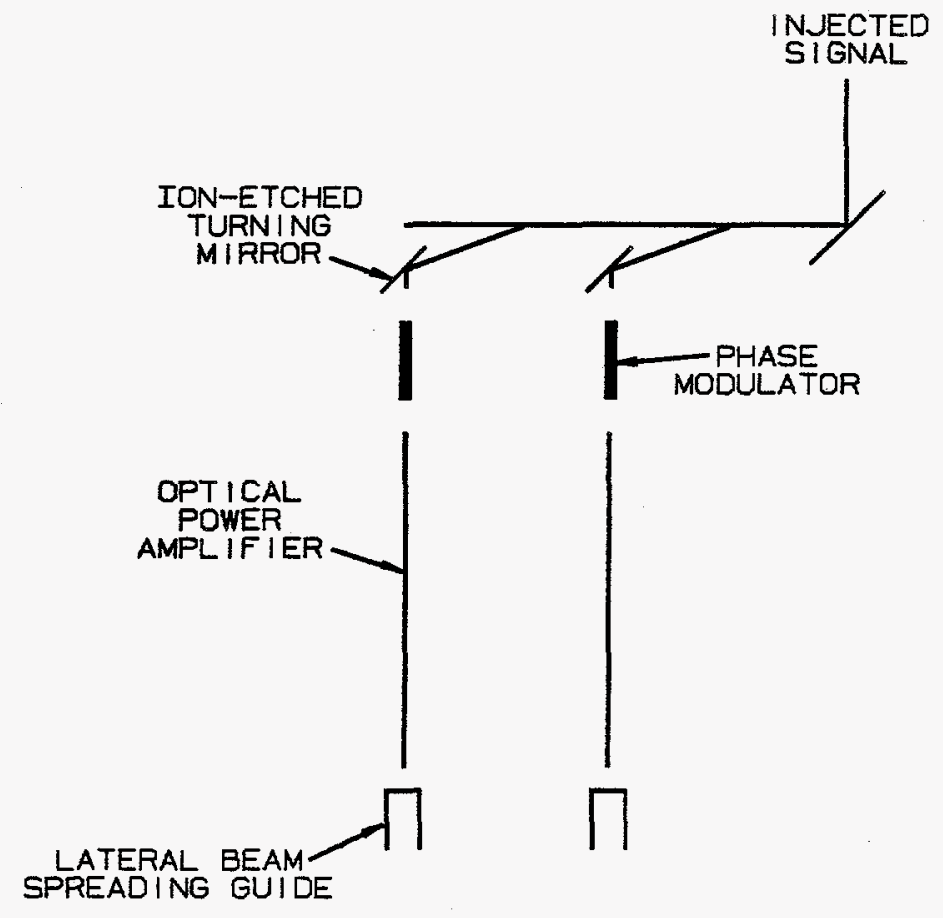

b. Block Diagram Showing Input and First Two Channels

Figure 10. Ten-Channel Phase Modulator and Amplifier Array Chip 


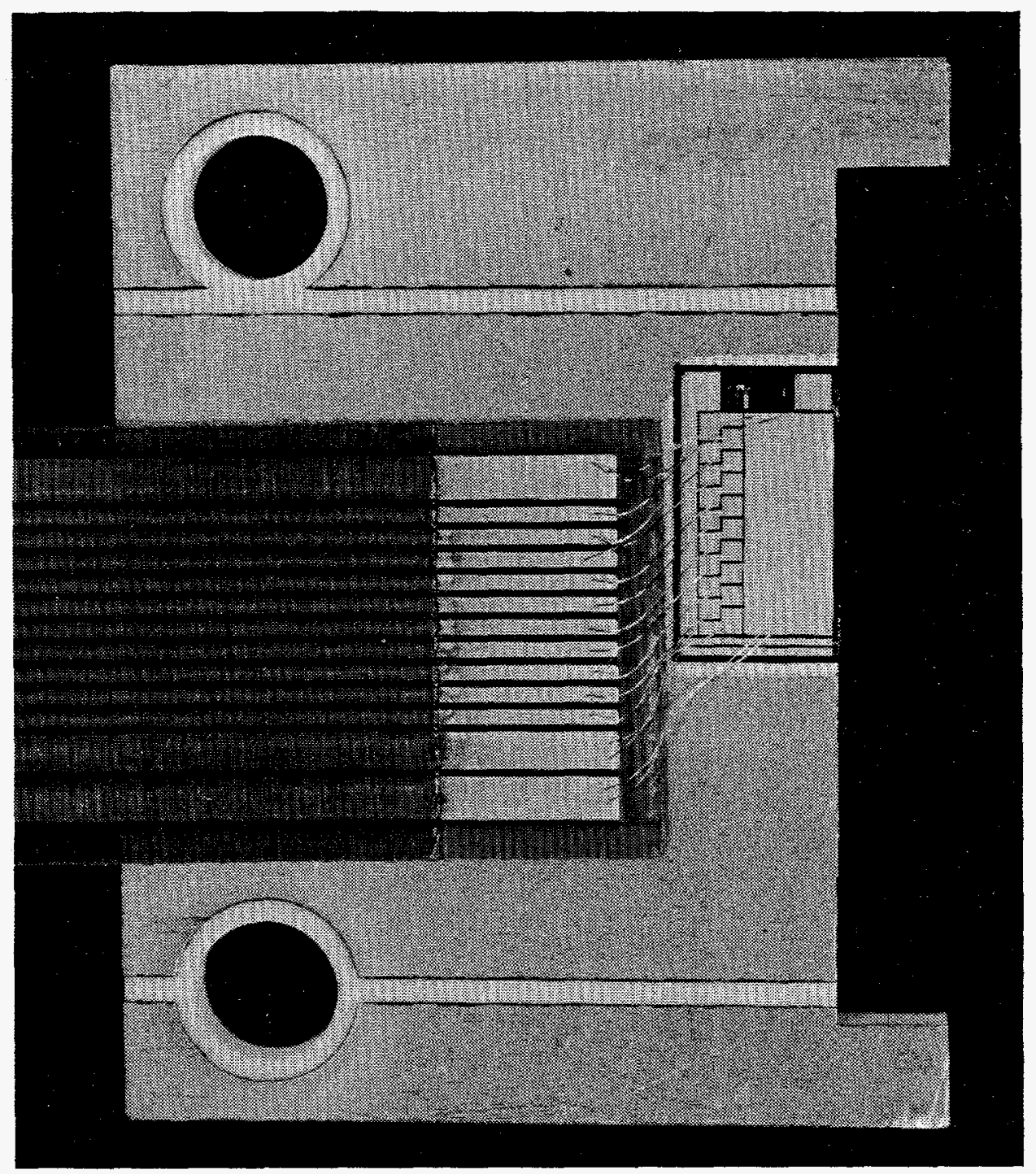

Figure 11. Laser Amplifier Array Packaged on BeO Substrate

were employed. Flat cable technology was employed for the electrical feeds; that was satisfactory for the original low-frequency application for this device. Satisfactory cooling of the device was achieved by using a copper block heat sink for the substrate.

\section{Repackaged Laser Amplifier Array}

A repackaged laser amplifier array is shown in Figure 12. This package was designed at KCD for high-speed operation. It includes microwave stripline electrical feeds with significantly shorter wire bond leads. Impedance-matching networks will be included on board the substrate this year. Coaxial lines are used for driving the striplines. Although the existing laser amplifier array is a low-frequency device, a redesign for higher frequency and higher power is being initiated for potential weapon applications. The type of packaging shown in Figure 12 is a first step for improving this device. Tapered fiber optic input will be attempted this year to improve input coupling. A silicon 


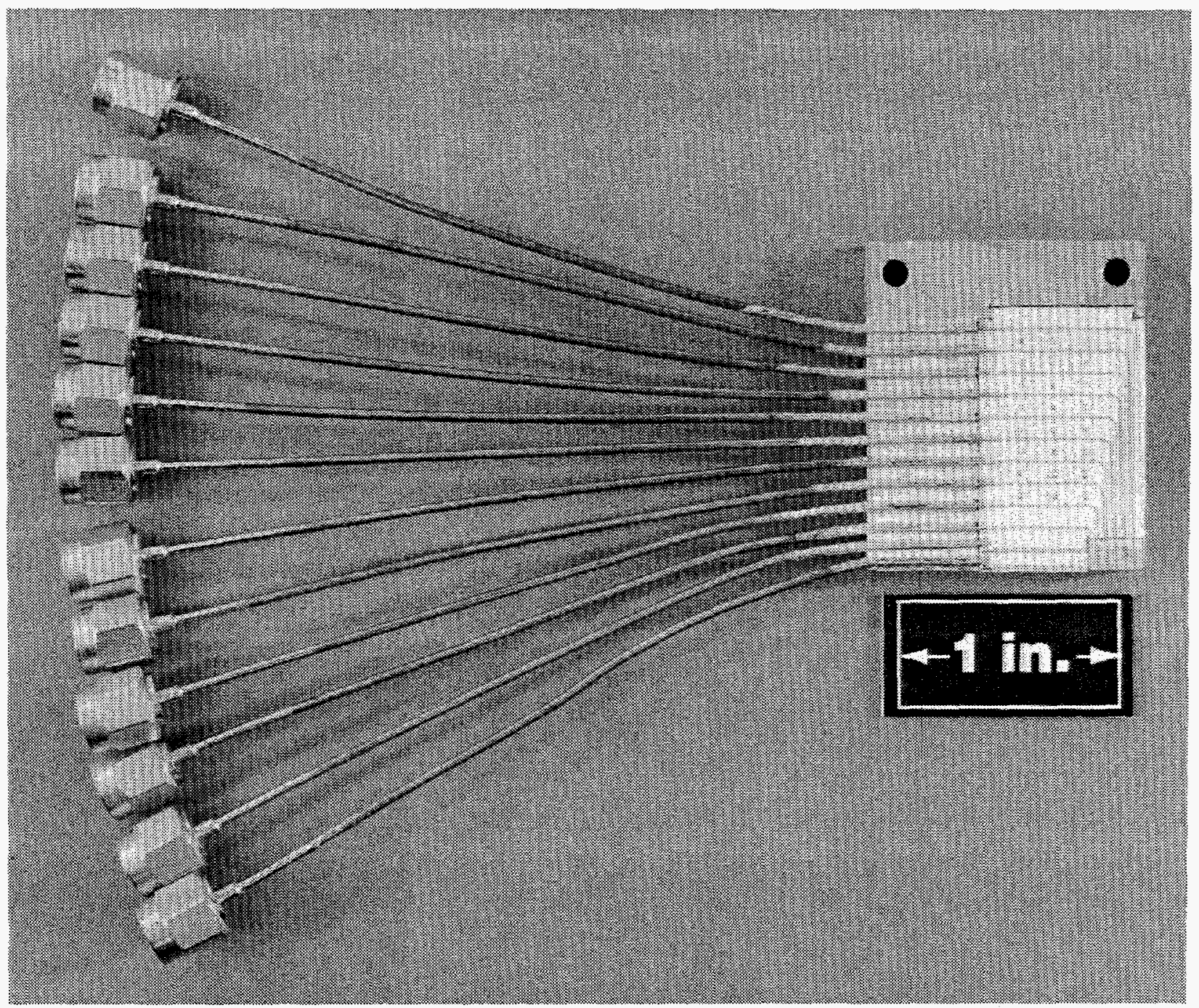

Figure 12. Repackaged Laser Amplifier Array

V-groove array will capture the 10 -channel output.

The input optical fiber is aligned on the right side of the package. Single-mode polarizing fiber is required, at the input, for operation of the laser amplifier array. The linear fiber array is aligned to the lower side of the package. The linear fiber array can be multimode. The laser amplifier array is a coherent device, however, and could employ single-mode or polarizing fiber at the output. Use of the laser amplifier array in a phased array radar system would require polarizing fiber output, for example. Currently, the fibers and fiber arrays are being aligned manually. A computer-controlled autoalignment microscope system is currently being purchased to facilitate the submicron alignment requirements of this chip.

Three designs of input fiber are being evaluated. Uncoated $3 \mathrm{M} \mathrm{PZ}$ polarizing fiber, mechanically tapered $3 \mathrm{M} \mathrm{PZ}$ polarizing fiber, and antireflection coated fibers are being evaluated. The primary concerns are system efficiency, laser diode feedback instability, and ease of alignment. All three fiber designs have tradeoffs in reliability, cost, and ease of 
alignment. Fiber soldering techniques are also being developed for fiber attachment.

A multimode silicon V-groove fiber optic array will be employed at the output to collect all 10 channels at once. Thermoelectric (TE) coolers will be employed to thermal cycle the package. Future applications of gallium arsenide electrooptic technology hinge on its ability to perform over the -65 to $165^{\circ} \mathrm{F}$ temperature range. Additional testing will be required to determine shock, vibration, and electromagnetic field (EMF) susceptibility.

Preliminary measurements of system efficiency, laser amplification, and modulation limits have been completed on the original chip mount shown in Figure 11. No TE cooling was employed for these initial measurements. The chip was mounted on a copper block heat sink and a polarized fiber input was aligned by mechanical staging. The test setup is shown in Figure 13. The redesigned package, Figure 12, will be extensively tested to determine the degree of improvement.

The test setup picture does not show the infrared microscope that facilitates alignment. The double stage of optical isolation and original source laser are also not visible in Figure 13. Newport 561 stages are used for fiber alignment. The copper block heat sink appeared to adequately cool the laser amplifier array, because no thermal instabilities were observed in chip output over time.

\section{Sandia Phase Modulator}

A phase modulator chip was furnished by Sandia and packaged at KCD (Figure 14). This device operates at 1.32 microns.

The tapered brass rods hold the input and output fiber optics. These fibers are aligned to the phase modulator waveguides by mechanical staging. This package was assembled to facilitate the alignment and programming of the computer-automated fiber alignment station, which is currently being purchased. This fiber alignment station includes computer control of input fiber, output fiber, and microscope head. A digital image processor has been assembled for macro alignment of fiber angle and polarization rotation alignment. The polarization rotation angle can be aligned by optical means or by digitizer measurement of fiber core eccentricity.

The phase modulator chip, Figure 14, is packaged in a Hewlett Packard modular microcircuit assembly. The chip is mounted between two microwave striplines in the HP package. Ten separate phase modulators are on this single chip. These modulator chips were originally manufactured by Sandia for the COMPASS project. Gold-plated ceramic ferrules were obtained from Kyocera Corp. for soldering the optical fibers through the package walls. Individual fibers have been gold plated at KCD, but plated fibers are now commercially available. The ceramic ferrule and gold-plated fibers are standard industry techniques for hermetically sealing packages. Although it is not an optimal solution, most commercial package designs use this approach.

\section{Optical Switch Design}

Optical switch arrays that can be employed as part of a programmable logic array are of great interest. A $2 \times 2$ optical switch, manufactured by AT\&T, is shown in Figure 15. This optical switch uses lithium niobate waveguide technology. The switch is capable of 500 megahertz operation. The two input optical signals either translate directly to the two optical outputs or they cross bar switch. The fiber optic attachments use silicon V-groove technology. This switch was bench tested at KCD. 


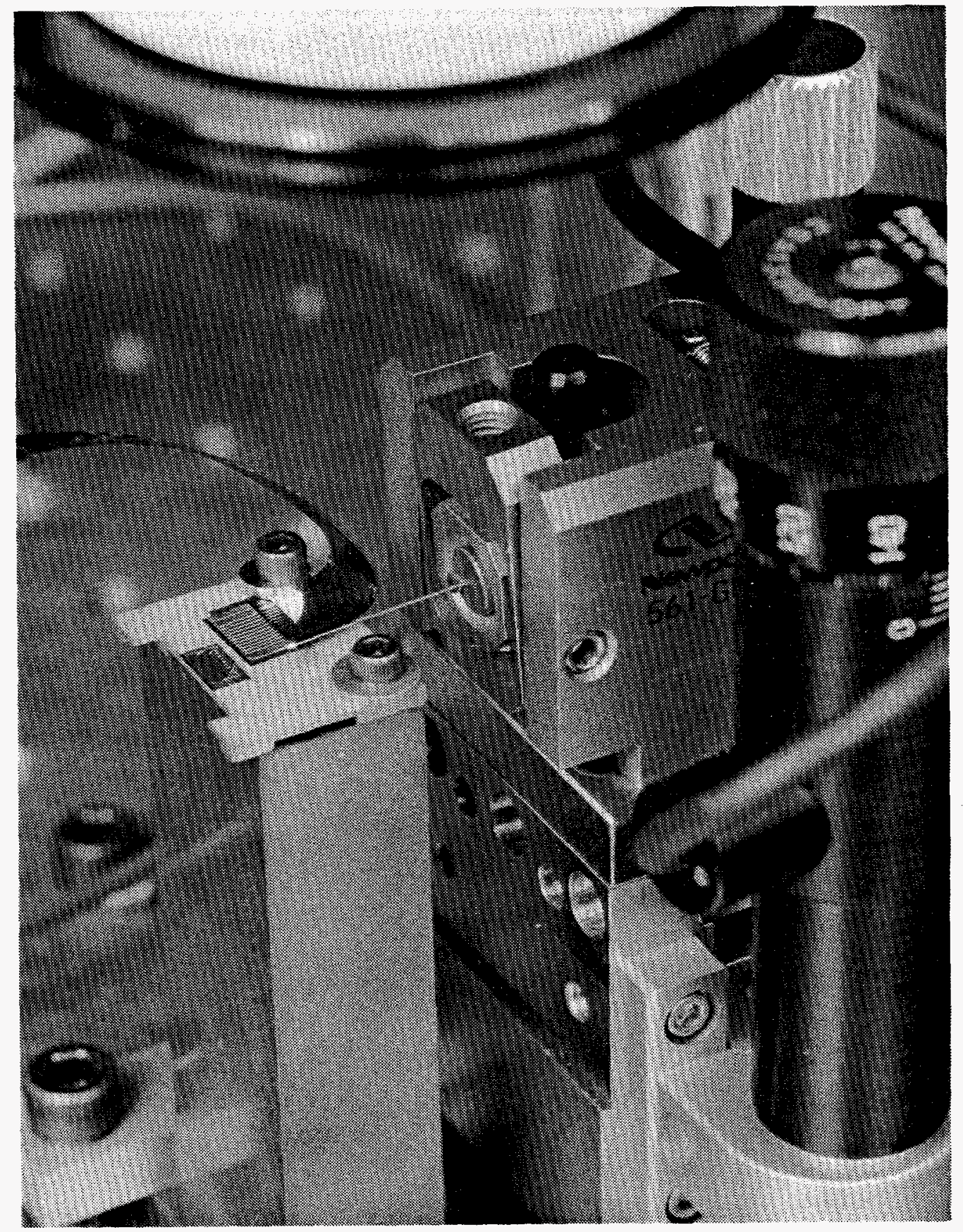

Figure 13. Laser Amplifier Array in Test Configuration

A packaging design capable of high speed operation over the -65 to $165^{\circ} \mathrm{F}$ temperature range is required if programmable logic arrays are to be used in weapon applications. A design study on three approaches to high speed switching using Mach-Zehnder electro-optic waveguides is included in the Appendix of this report. A design study on a packaging approach capable of operating over the -65 to $165^{\circ} \mathrm{F}$ temperature range has been initiated. These designs will use lithium niobate waveguide technology. 


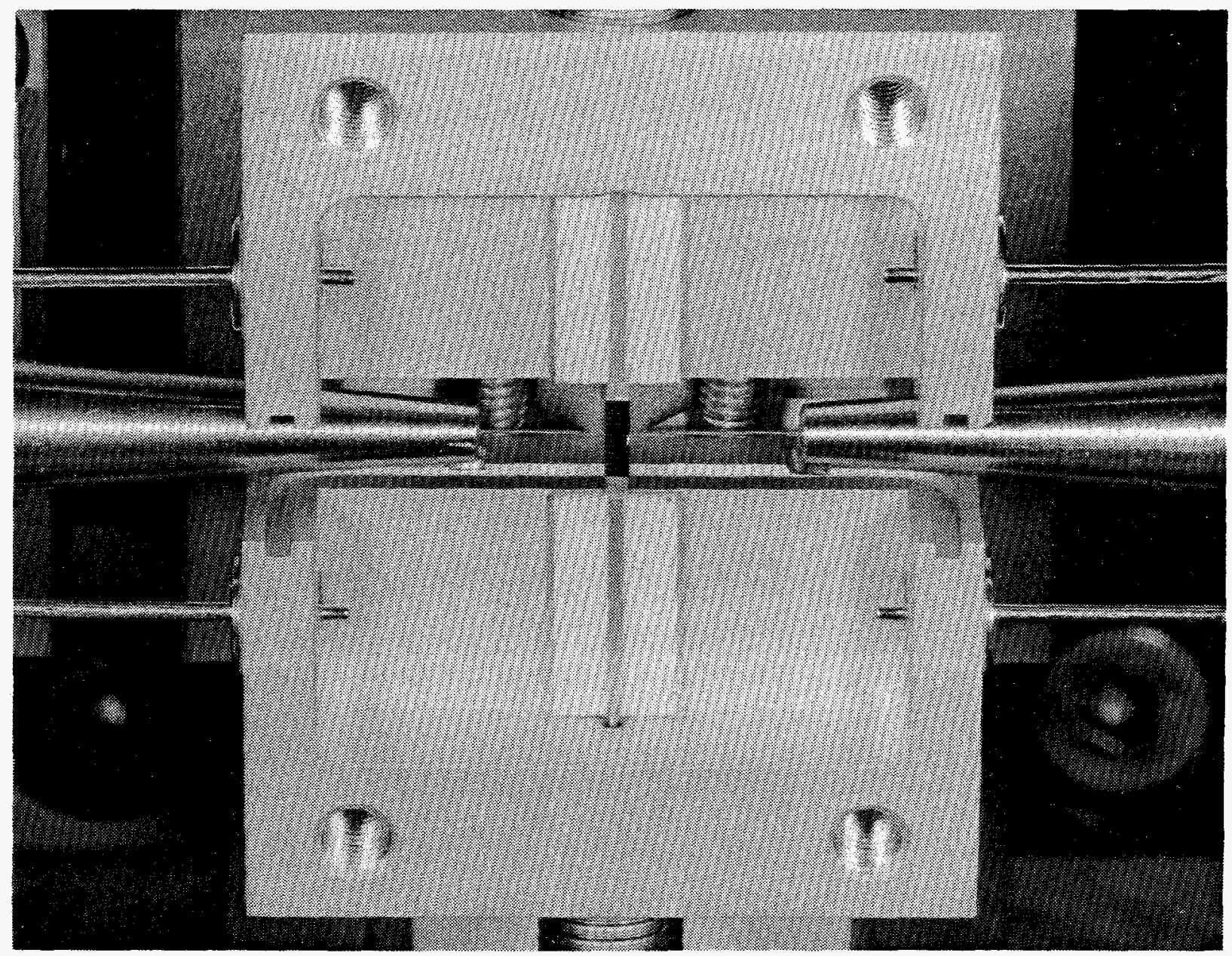

Figure 14. Packaged Sandia Phase Modulator

\section{Accomplishments}

Major packaging challenges have been overcome in this project. Polarization fiber optic alignment has been achieved.

Conventional lens arrays have been used to efficiently couple polarization fiber optics to gallium arsenide waveguides. Gallium arsenide electro-optic integrated circuits have been acquired, die attached in packages, and wire bonded.

Manufacturing processes for fiber optics have been exercised. Fiber optics have been chrome/gold plated, optically aligned to submicron precision, and eutectically soldered to crystalline silicon V-groove arrays to form a rugged linear fiber optic array capable of withstanding -65 to $165^{\circ} \mathrm{F}$ temperature environments.

Preliminary characterization of a laser amplifier and modulator array has been accomplished. The gallium arsenide array has been repackaged for higher speed operation. Modifications of the laser amplifier array are being identified to increase power and modulation speed for potential KCD WR applications.

A $36 \times 36$ microlens array has been aligned and attached to a 15-fiber linear $V$-groove array and delivered to Sandia for testing with advanced gallium arsenide chip designs. Custom positioning 


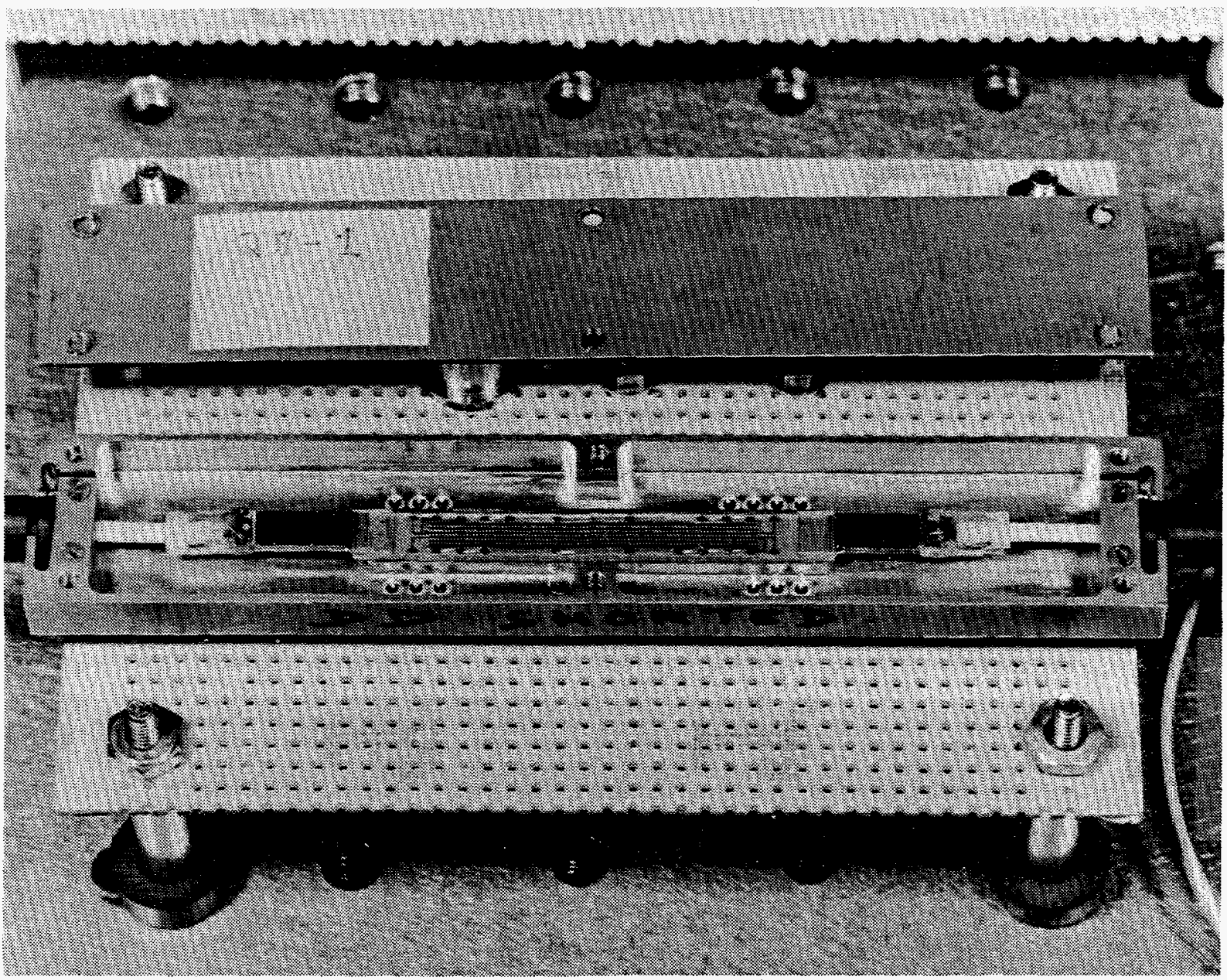

a. Optical Switch

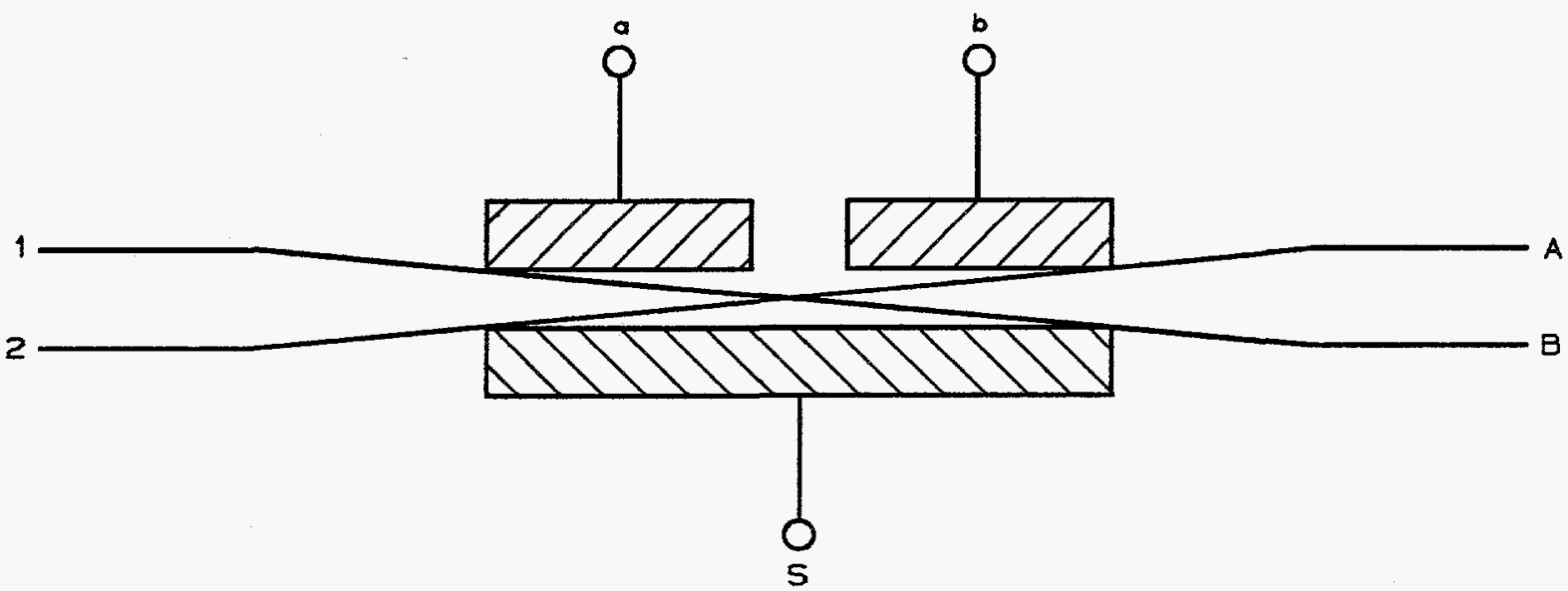

b. Optical Signal Inputs and Outputs

Figure 15. $2 \times 2$ Lithium Niobate Optical Switch by AT\&T 
equipment was fabricated at KCD for this assembly.

A Sandia-supplied gallium arsenide phase modulator has been packaged for testing the new computer-controlled microscope fiber alignment station. This system is currently on order.

A design study of lithium niobate switches was initiated. The goal is to achieve a design that can be used in a logical switch array and that will operate over the -65 to $165^{\circ} \mathrm{F}$ temperature range. Both device and packaging requirements are being studied. This study will identify contenders for an optical logic array for potential KCD WR applications.

Fabrication methods for a two-dimensional fiber optic array are being investigated. These include laser drilling of ceramic, etched quartz substrates, and stacked twodimensional $V$-groove arrays.

\section{Future Work}

Flip chip bonding and silicon bench technology are essential processing technologies that need to be developed at KCD. The future KCD work in electro-optic integrated hybrid circuits will require these technologies.

A computer-controlled microscope alignment station is currently being funded. This station will employ an infrared microscope and detector system to aid in device pigtailing, lens array alignment, and device characterization. This system has been custom engineered to provide computer autoalign of fiber and waveguide, image processor measurement of polarization fiber rotation angle, and waveguide mode structure analysis.

Significant help on the design of this station was provided by Sandia personnel. The autoalign, image processor polarization analyzer, and waveguide mode analyzer are being engineered at KCD. This microscope system will also be used in planar lens array alignment studies.

The 10-channel laser amplifier array will be packaged and characterized this year. The packaging approach for this device will be applicable to future higher speed designs.

A specific lithium niobate Mach-Zehnder based switch will be packaged for -65 to $165^{\circ} \mathrm{F}$ temperature range evaluation.

Multiple fiber alignment techniques will be explored. Commercial equipment will be reviewed for welding or soldering fibers to hybrid assemblies. 


\section{Reference}

${ }^{1}$ S.S. Ou, et al, TRW, Research Center, Space \& Technology Group. “High Performance Surface-Emitting Lasers With 45 Degree Intracavity Micromirrors." Appl. Phys., Lett. 58 (1), January 7, 1991. 



\section{Appendix}

\section{Design Review of the Integrated Optical Maze}

By R. A. Becker 


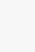




\section{Contents}

Section Page

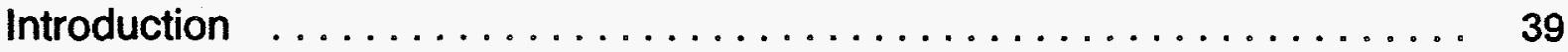

Integrated Optical Maze Architecture $\ldots \ldots \ldots \ldots \ldots \ldots \ldots$

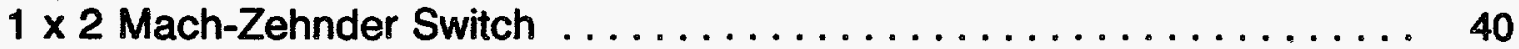

$2 \times 2$ Mach-Zehnder Switch $\ldots \ldots \ldots \ldots \ldots \ldots \ldots \ldots \ldots \ldots \ldots \ldots \ldots$

$2 \times 2$ Directional Coupler Switch $\ldots \ldots \ldots \ldots \ldots \ldots \ldots \ldots \ldots \ldots \ldots$

Design Aspects Discussion $\ldots \ldots \ldots \ldots \ldots \ldots \ldots \ldots \ldots \ldots \ldots \ldots \ldots \ldots$

Optical Output Power, Wavelength, and Pulse Length $\ldots \ldots \ldots \ldots \ldots$

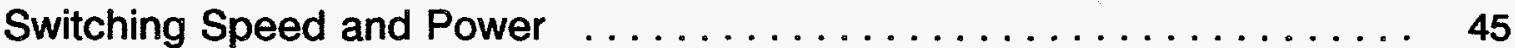

Number of Switching Nodes $\ldots \ldots \ldots \ldots \ldots \ldots \ldots \ldots \ldots \ldots$

Coupling Loss In/Out of the $10 M \ldots \ldots \ldots \ldots \ldots$

Loss Per Switching Node $\ldots \ldots \ldots \ldots \ldots \ldots \ldots$

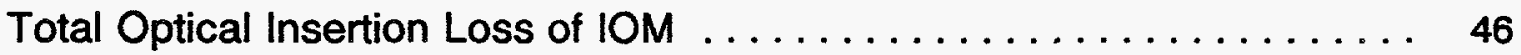

Switching Voltage and Current $\ldots \ldots \ldots \ldots \ldots \ldots \ldots \ldots \ldots \ldots$

Physical Size and Weight $\ldots \ldots \ldots \ldots \ldots \ldots \ldots \ldots \ldots \ldots \ldots \ldots$

Optical Source Characteristics $\ldots \ldots \ldots \ldots \ldots \ldots \ldots \ldots \ldots \ldots \ldots$

IOM Material System $\ldots \ldots \ldots \ldots \ldots \ldots \ldots \ldots \ldots \ldots \ldots \ldots \ldots$

Optical Damage and Ferroelectric Waveguide Devices . . . . . . . . . . 49

Integrated Optical Maze Design $\ldots \ldots \ldots \ldots \ldots \ldots$

Summary and Conclusions $\ldots \ldots \ldots \ldots \ldots \ldots \ldots$ 


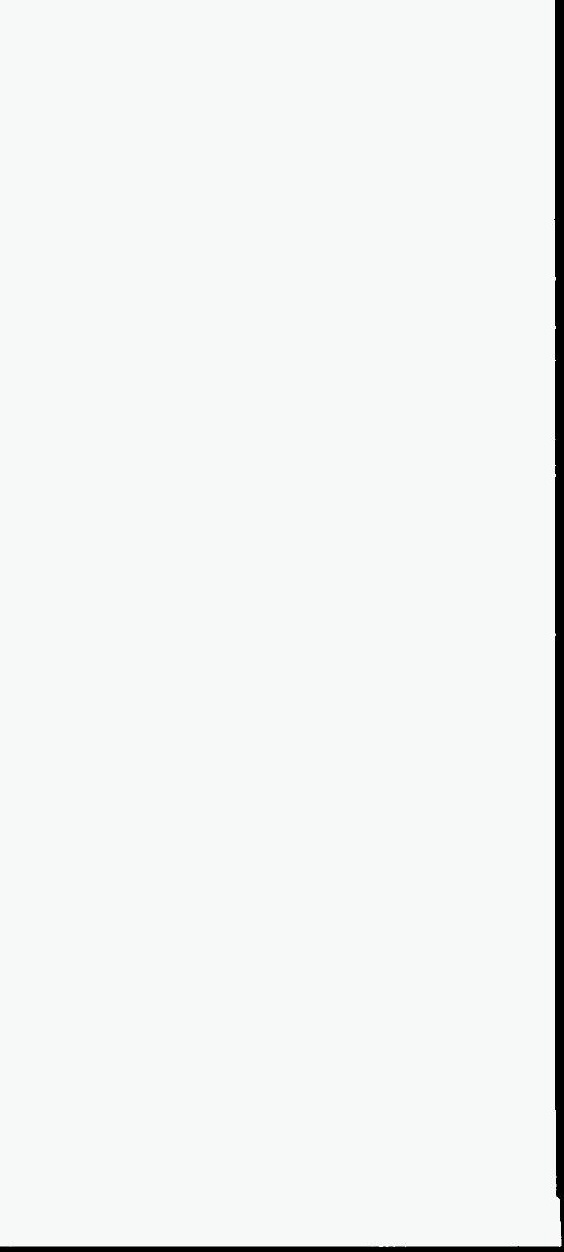




\section{Illustrations}

Figure $\quad$ Page

A-1 Four-Node Integrated Optical Maze Schematic . . . . . . . . . . . 40

A-2 $1 \times 2$ Mach-Zehnder Switch $\ldots \ldots \ldots \ldots \ldots \ldots \ldots \ldots \ldots \ldots \ldots \ldots$

A-3 $2 \times 2$ Mach-Zehnder Switch $\ldots \ldots \ldots \ldots \ldots \ldots \ldots \ldots \ldots \ldots$

A-4 $2 \times 2$ Directional Coupler Switch $\ldots \ldots \ldots \ldots \ldots \ldots \ldots \ldots$

A-5 Sixteen-Node Integrated Optical Maze Schematic $\ldots \ldots \ldots \ldots . \ldots . . .47$

A-6 Photorefractive Time Constant Versus Optical Power in IOM ... . . . 52

A-7 Design of IOM Switch Node $\ldots \ldots \ldots \ldots \ldots \ldots$

A-8 Four-Node Integrated Optical Maze Design $\ldots \ldots \ldots \ldots \ldots \ldots$

\section{Tables}

Number

Page

A-1 Insertion Loss of IOM for Different Material Systems . . . . . . . . . 47

A-2 Power Handling Capabilities $\ldots \ldots \ldots \ldots \ldots \ldots \ldots \ldots \ldots$

A-3 IOM Design Specifications $\ldots \ldots \ldots \ldots \ldots \ldots \ldots \ldots \ldots \ldots$ 


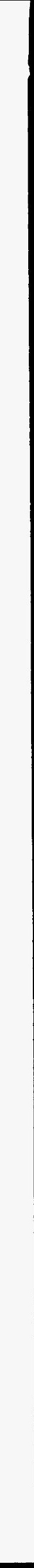




\section{Introduction}

In this document a design review of a concept known as the integrated optical maze (IOM) is presented. The IOM consists of a series of electronically, or optically, addressable optical switches configured in such a way that optical transmission through the IOM will occur if and only if a proper address sequence is input to the device. In addition, the IOM must be robust against optical transmission resulting from catastrophic damage. The IOM must be capable of routing a $650 \mathrm{~mW}, 10-\mathrm{ms}$ long optical pulse in the near-infrared (0.8 to $1.06 \mu \mathrm{m})$ wavelength regime.

Aspects of the IOM design that are viewed as important to the mission include:
A. Optical Output Power
B. Optical Pulse Length
C. Switching Speed
D. Optical Wavelength
E. Number of Switching Nodes
F. Coupling Loss In and Out of the IOM
G. Loss per Switching Node
H. Total Optical Insertion Loss of IOM

I. Switching Voltage and Current

J. Physical Size and Weight

K. Optical Source Characteristics

L. IOM Material System.

In this design review, an architecture is first proposed that satisfies the global requirements of the IOM. Next, this architecture is investigated with respect to the specific requirements listed above. Various simple examples of the flexibility of the approach are presented and problem areas are identified. Finally, a material system is chosen and a design is developed to demonstrate the feasibility of the IOM concept.

\section{Integrated Optical Maze Architecture}

The IOM will require a pulsed optical source, an electronically or optically addressable optical switching array, and control logic circuitry. The optical output of the IOM may go directly to the next stage or can first be amplified in a semiconductor optical amplifier (SOA).

Consider the optical switching arrangement depicted in Figure A-1. Here a series of four switching nodes are optically connected in some (currently) undetermined fashion. The single output port goes to the next stage of the system; the four test ports are used as inputs to the control circuitry. One possible arrangement would use two separate lasers: a high-power laser input to the primary optical input (POI) and a lowpower laser input to the secondary optical input (SOI).

Let us assume that a "Red" condition results in the high-power input pulse being routed to the IOM output. For both security and safety reasons, a series of low-power pulses can be routed from the SOI through the IOM before the onset of any Red condition. A predetermined, or continually programmable, address code could be used to both "Test" and "Ready" the IOM logic circuitry.

Only after all of the logic states have been satisfied can a Red condition be implemented. In addition, the logic circuitry could be arranged so that any abnormality that occurred during the Test and Ready phases would cause the system to go to a "Lock Up" state in which the high-power laser would not be functional and therefore, a high-power optical pulse could not be generated. This Lock Up state could be permanent 


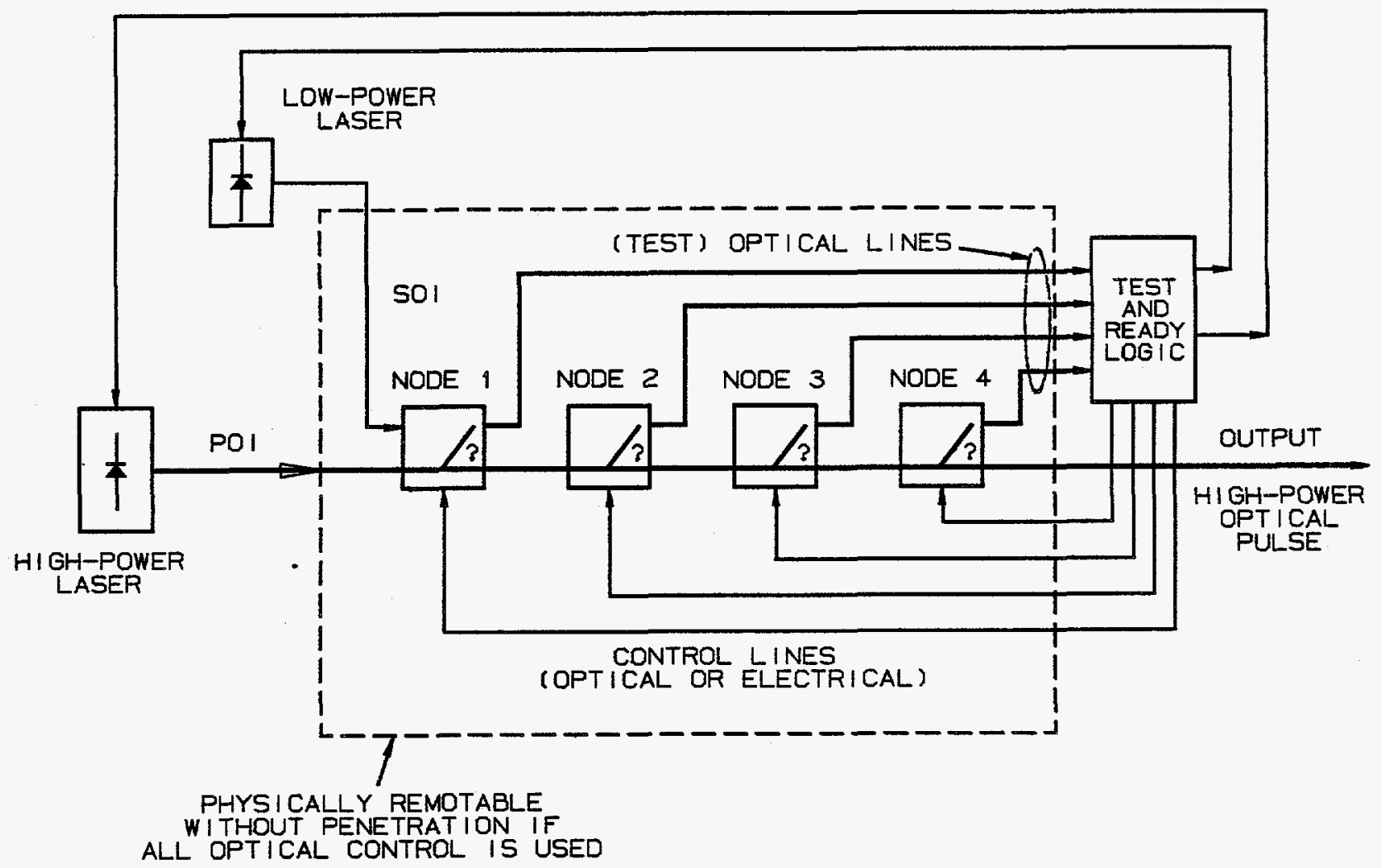

Figure A-1. Four-Node Integrated Optical Maze Schematic

(i.e., destruction of the high-power laser), requiring physical replacement, or could be temporary, requiring the onset of another Test and Ready sequence.

The individual switching nodes could be fixed or continually programmable with either electrical or optical control signals. Thus, the application of a control "High" could sometimes turn the switch to its "On" state and sometimes turn the switch to its "Off" state, depending on how the node had been programmed.

Obviously, the most important part of the IOM is the choice of optical switching node. Several possibilities exist and are reviewed here. The primary constraint on the switching node is that it have at least one input and at least two outputs. Three switching nodes satisfy these requirements:
A. $1 \times 2$ Mach-Zehnder Switch,
B. $2 \times 2$ Mach-Zehnder Switch, and

C. $2 \times 2$ Directional Coupler Switch.

\section{$1 \times 2$ Mach-Zehnder Switch}

An optical intensity modulator, or switch, can be constructed by combining a passive Y-splitter with a phase modulator and a passive 3-dB directional coupler as shown in Figure A-2a. In this device, the incoming light is split into two equal components by the input $Y$-splitter. A differential phase shift is then imparted by applying an electrical signal to the differential phase modulator electrodes. Finally, the two light components interact in the output $3-d B$ coupler. If no signal is applied, then both outputs have the same intensity; if a $-\pi / 2$ phase shift is applied (i.e., $V=-V_{\pi} / 2$ ), all the light exits the top output port; if $a+\pi / 2$ phase shift is applied (i.e., $V=+V_{\pi} / 2$ ), all the light exits the bottom output port. An expression for the voltage-in/light-out transfer function for this switch is given by 


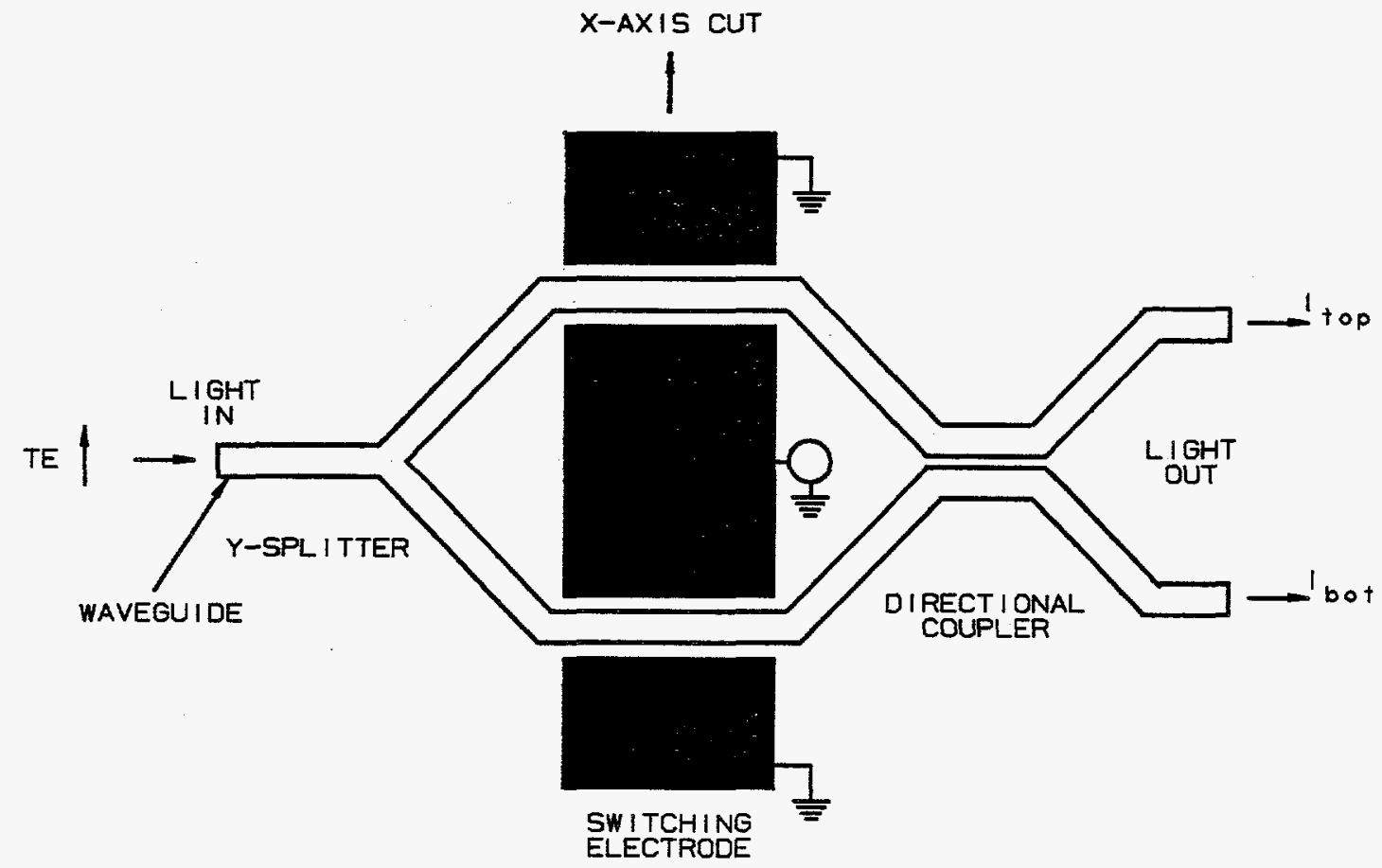

a. Switch Schematic

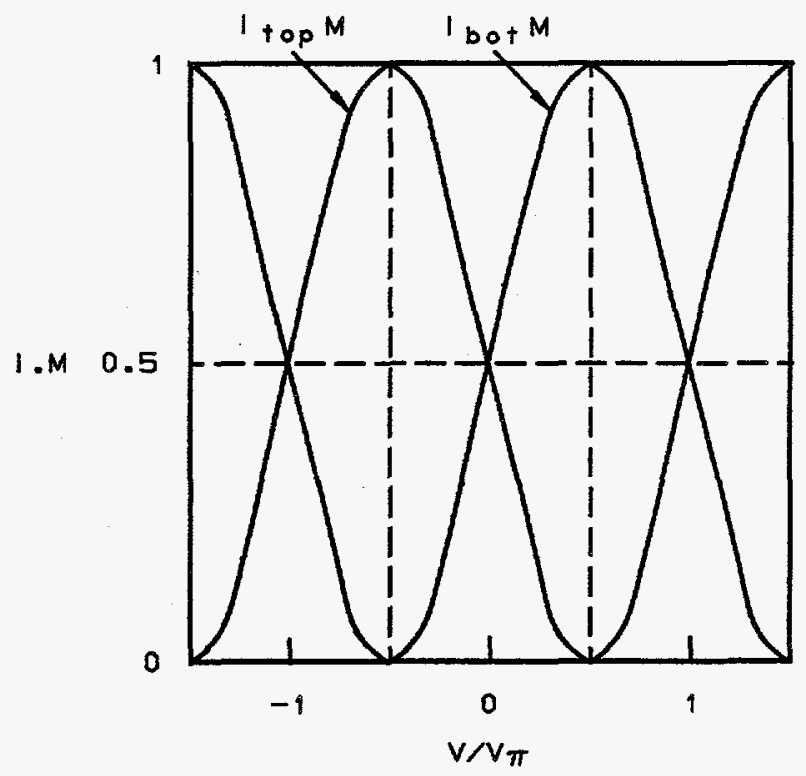

b. Light OutNoltage In Transfer Function

Figure A-2. $1 \times 2$ Mach-Zehnder Switch

$$
\begin{aligned}
& I_{\text {top }}=I_{0} \cos ^{2}\left(\pi V / 2 V_{\pi}+\pi / 4\right), \\
& I_{\text {bot }}=I_{0} \sin ^{2}\left(\pi V / 2 V_{\pi}+\pi / 4\right),
\end{aligned}
$$

and is shown schematically in Figure A-2b. The main drawback of this type of switch for the $10 \mathrm{M}$ is the fact that for no applied voltage, a partial (1/2) transmission occurs. This could interfere with the requirement that the device be robust with respect to various stimuli. 


\section{$2 \times 2$ Mach-Zehnder Switch}

A variation of the $1 \times 2$ Mach-Zehnder switch which replaces the input $Y$-splitter with a passive 3-dB directional coupler takes care of the above deficiency. This arrangement is shown in Figure A-3a. In this switch, the input 3-dB directional coupler splits the input light evenly but imparts a $\pi / 2$ phase shift between the two branches. The output 3-dB directional coupler once again allows the light from the two paths to interact coherently.

If no voltage was applied, then all the light that was incident on the top input port would exit the bottom port and the switch would be in the "Cross" state. If a $\pi$-phase shift is introduced through the application of voltage (i.e., $V=+V_{\pi}$ ), then all the light exits from the top port and the switch would be in the "Bar" state.

An expression for the voltage-in/light-out transfer function for this switch when the top port is excited optically is given by

$$
\begin{aligned}
& I_{\text {top }}=I_{0} \cos ^{2}\left(\pi V / 2 V_{\pi}+\pi / 2\right), \\
& I_{\text {bot }}=I_{0} \sin ^{2}(\pi V / 2 V \pi+\pi / 2),
\end{aligned}
$$

and is shown schematically in Figure A-3b. This switch has the beneficial attribute that it can always be in the Off or Cross state without applied voltage.

\section{$2 \times 2$ Directional Coupler Switch}

The final switch to be considered is the classical directional coupler switch. This switch, which is shown in Figure A-4a, consists of an active directional coupler and is similar to the previously discussed $2 \times 2$ Mach-Zehnder switch. In this switch, both the amplitude and phase of the light in the active coupler region are altered by an applied voltage. By choosing the coupler to be one coupling-length long, light input to the top port will exit the bottom port when no voltage is applied. The voltage-in/lightout transfer function of the directional coupler switch for light input to the top port and a one-coupling-length long coupler is given by the expression

$$
\begin{aligned}
I_{\text {top }}= & \sin ^{2} K L^{*} \text { sqrt }\left[1+(\Delta \beta / 2 K)^{2}\right] / \\
& {\left[1+(\Delta \beta / 2 K)^{2}\right], } \\
I_{\text {bot }}= & 1-I_{\text {top }},
\end{aligned}
$$

and is shown in Figure $A-4 b$. Here, $k$ is the coupling constant and $\Delta \beta$ is the voltageinduced change in the propagation constants of the two waveguides. The primary functional difference between the directional coupler switch and the $2 \times 2$ Mach-Zehnder switch is that the latter has a periodic transfer function and the former does not. From a practical standpoint, one can be more easily fabricated in some material systems than the other and vice versa.

All of the switches discussed here can be fabricated or externally controlled to be in whatever state is desired, although some are more easily fabricated in one state or the other or midway in between, as was discussed. For the IOM, an Off state without an applied input would seem to be advantageous to guard against undesired Red conditions. Thus, the latter two switch types appear to be the most applicable to the IOM.

Although any of the switches can be fabricated to be in a particular state, after fabrication they can also be electrically or optically biased to be in any other state; thus, they are programmable. For example, consider the $2 \times 2$ Mach-Zehnder switch depicted in Figure A-3. If no programming voltage is applied, then the switch will be in the Off or Cross state. However, if a programming voltage of $V_{\pi}$ is applied, then the switch resides in the On or Bar state and an externally applied voltage would be required to turn the switch off.

More than one set of electrodes could be used on the switch: a programming 


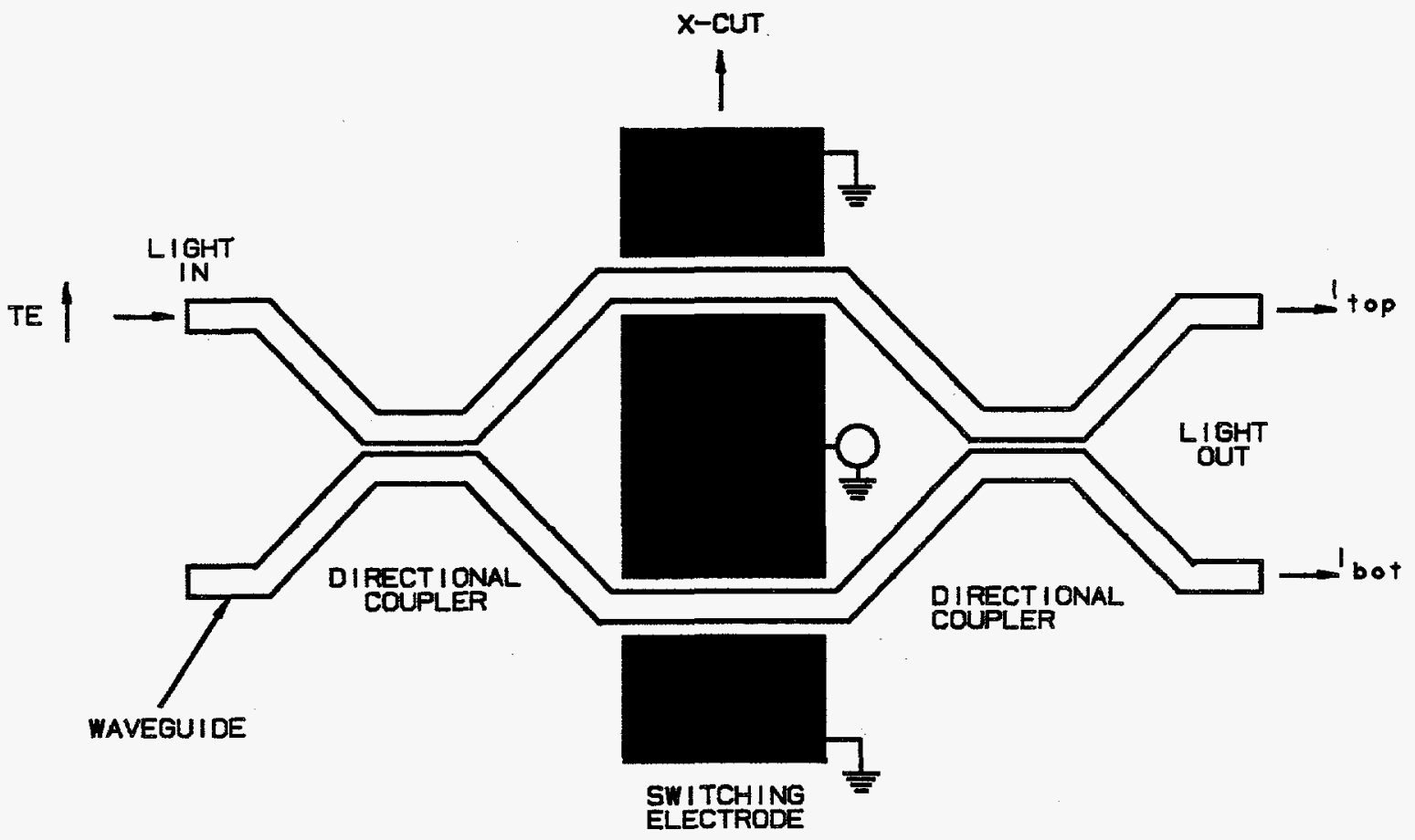

a. Switch Schematic

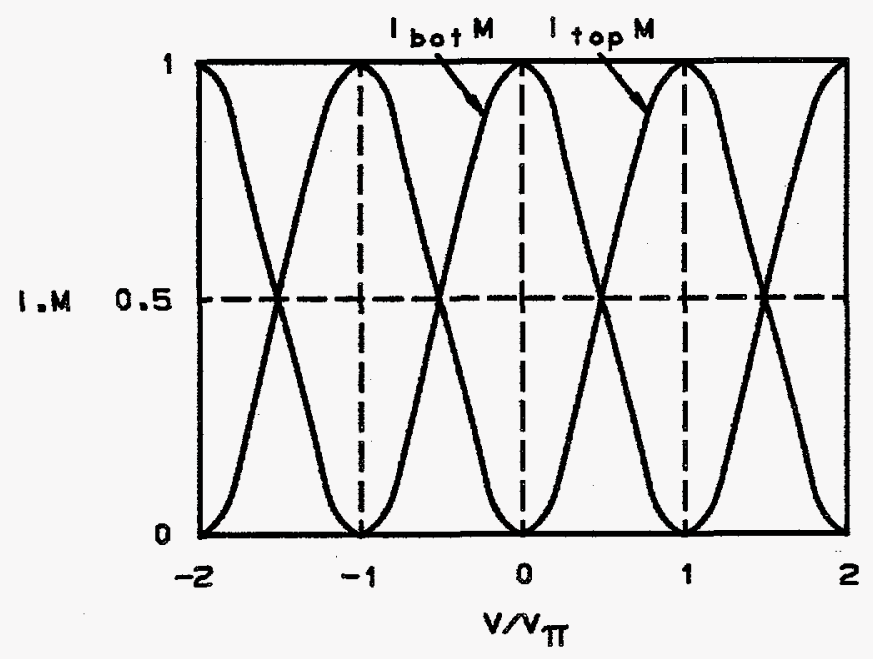

b. Light OutVoltage In Transfer Function

Figure A-3. $2 \times 2$ Mach-Zehnder Switch

electrode and a control electrode, for example. Thus, the set of voltages required to bring the IOM to the Red condition could be an ever-changing code. One can think of it as a combination lock where the combination continually changes; one must know the correct combination at the given instant a Red condition is desired in order for the lock to open. A combination of normally on and normally off states would provide the best protection against a false Red condition occurring.

Up to this point, electrical control voltages were assumed. However, to switch these 


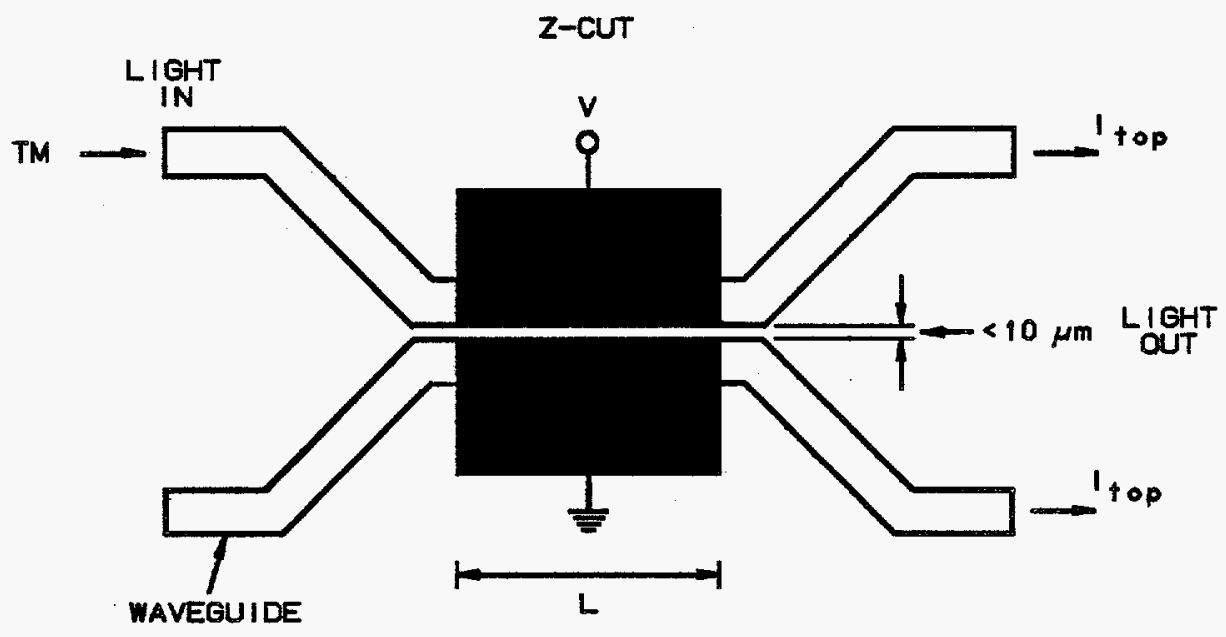

a. Switch Schematic

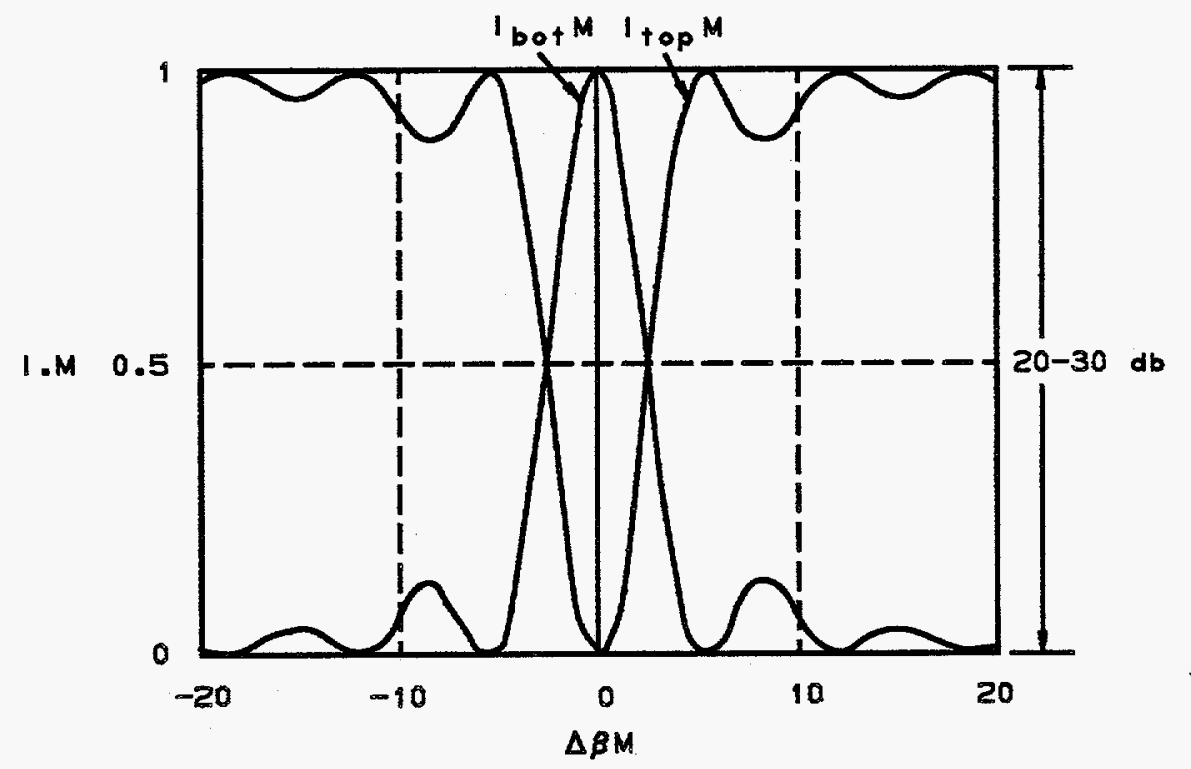

b. Light Out/Voltage In Transfer Function

Figure A-4. $2 \times 2$ Directional Coupler Switch

nodes, very little power is required because they are voltage-actuated devices. The only real current that needs to be supplied is that required to overcome the device leakage which is in the pA to $n A$ range. Thus, by using a photocell and a 1 to $10 \mathrm{k} \Omega$ resistor in parallel with the device electrodes, the switches could be turned on and off using light pulses.

Because the switch capacitance is typically less than $10 \mathrm{pF}$, the resistance capacitance (RC) time constant for even a $10-k \Omega$ resistor would still allow the desired $1-\mathrm{ms}$ rise and fall times.

In summary, the architecture depicted in Figure A-1 appears to be a good candidate for the IOM. It provides a very flexible approach and is compatible with existing switch architectures. 


\section{Design Aspects Discussion}

In this section, each of the critical design aspects of the IOM are discussed with respect to the architecture discussed above and depicted in Figure A-1.

\section{Optical Output Power, Wavelength, and Pulse Length}

A 10-ms wide, 650-mW peak-optical-power pulse is required as the input to the next stage of the system in which the IOM will be used. Two options exist for delivering this amount of optical power:

A. Provide sufficient optical power to the input of the IOM so that $650 \mathrm{~mW}$ is available at the output of the IOM, or

B. Follow the output of the IOM with a semiconductor optical amplifier (SOA).

Option A requires the use of a laser capable of producing a 10-ms wide optical pulse in the 0.8- to $1.0-\mu \mathrm{m}$ spectral range, with a preferably Gaussian beam profile capable of being focused to a nearly diffraction limited spot for input to the IOM. Working backwards, assuming IOM losses (including input and output coupling losses) of about $6 \mathrm{~dB}$, it is clear that the laser would have to have a peak output of over $2.5 \mathrm{~W}$. This is currently not possible using semiconductor diode lasers.

The highest power commercially available semiconductor laser that meets the above requirements is only capable of producing $1 \mathrm{~W}$ continuous power (CW) at $0.985 \mu \mathrm{m}$ and is available from Spectra Diode Laboratories. This device includes a monolithicly integrated master oscillator/power amplifier (MOPA) structure to provide the 1-W output. Approximately 3 amps of drive current are required by the power amplifier, but the device is capable of being modulated at rates up to $20 \mathrm{MHz}$ by modulating the $120-\mathrm{mA}$ drive current of the oscillator. Although higher pulsed or CW output may be available from diode lasers, these are achieved by using multiple stripes, whose collective outputs are not easily focused to a diffraction limited spot.

Option B, on the other hand, would allow a lower power laser to be used as the input to the IOM. Then, both the original lowpower level and the IOM loss could be compensated for with an SOA following the IOM. For example, the power amplifier present in the Spectra Diode Lab device could be used as the post-IOM SOA. Assuming an SOA with 10-dB gain and an IOM with 6-dB total loss, the input diode laser would only need to produce $260 \mathrm{~mW}$ of output.

Because of the recent interest in the $0.985-\mu \mathrm{m}$ region of the spectrum for diodepumped solid-state (DPSS) laser pump sources, these high-power semiconductor lasers have been rapidly developed, and reliability performance has been carefully scrutinized. For this reason, the use of a $0.985-\mu \mathrm{m}$ wavelength seems preferable over the use of a 0.8- $\mu \mathrm{m}$ wavelength. In addition, the longer wavelength is less likely to cause optical damage effects that occur in dielectric waveguide materials like $\mathrm{LiNbO}_{3}$ and $\mathrm{LiTaO}_{3}$; the longer wavelength is within the transparency range of GaAs waveguides if one desired to base the IOM on a semiconductor. The use of an SOA at the IOM output also appears to be a preferred approach.

\section{Switching Speed and Power}

The switching speed of integrated optical switches that operate through the electrooptic effect is inherently fast, limited by the RC time-constant of lumped-element electrodes or the velocity mismatch constant for traveling-wave electrodes. Typical devices have capacitances of less than $1 \mathrm{pF} / \mathrm{mm}$ of electrode length.

Because the overall size of the IOM must be less than the $76-\mathrm{mm}$ diameter wafer 
size, even with a $10-\mathrm{k} \Omega$ load resistor, the $1-\mathrm{ms}$ rise time of the switch is not impeded. The switching power required is primarily determined by the size of the resistor used, if any. For example, a 10-V switching voltage and a $10-\mathrm{k} \Omega$ resistor would require $10 \mathrm{~mW}$ of electrical power to switch.

There also exist thermally operated switches which are built on nonelectrooptic materials such as glass on silicon. These switches are polarization independent, can handle large optical powers, and can have rise times $<1 \mathrm{~ms}$. Given that their switching speed is acceptable for the application, their biggest drawback is the switching power required which is on the order of $1 / 2 \mathrm{~W}$ per switch (Takato, et. al., SPIE, Vol. 1177, 92, 1989).

\section{Number of Switching Nodes}

The number of switching nodes is determined by the switch size, the substrate size, and the number of interconnected substrates. Although there is a limit on the number of serially connected switches that one can fabricate on a given length of substrate, an architecture as shown in Figure A-5 can greatly increase the total number of switching nodes. Here, the same basic architecture as shown in Figure $A-1$ is repeated four times, being interconnected with optical fiber. This is possible because integrated optical switches tend to be much longer (10's of $\mathrm{mm}$ ) than they are wide (0.1's of $\mathrm{mm}$ ). Thus, an architecture like that shown in Figure A-1 that contains four nodes could easily be extended to 16 or 32 nodes without much difficulty.

\section{Coupling Loss In/Out of the IOM}

Assuming that the $10 \mathrm{M}$ is fiber pigtailed, the primary input/output coupling loss of the IOM is determined by the mode mismatch loss between the fiber and the $1 O M$ and their positioning with respect to each other. In glass waveguides (such as thermo-optic switches), the loss can be as low as $0.5 \mathrm{~dB} /$ interface or better. In devices based on $\mathrm{LiNbO}_{3}$ or $\mathrm{LiTaO}_{3}$, less that $1 \mathrm{~dB} /$ interface is typical. For devices based on semiconductors, such as GaAs, typical losses are in the 2 to $3 \mathrm{~dB}$ /interface range, primarily because of the poor mode match between the semiconductor waveguide and the optical fiber. These high interface losses become very important when interconnected architectures like that shown in Figure A-5 are considered.

\section{Loss Per Switching Node}

The loss per switching node is largely dependent on the material system and the switch design. In glass (thermo-optic switch) the loss per node could be considerably less than $1 \mathrm{~dB}$ because of the relatively high-index waveguides and the extremely low propagation loss. In materials like $\mathrm{LiNbO}_{3}$ and $\mathrm{LiTaO}_{3}$, the losses will be somewhat higher but should not exceed 1 to $2 \mathrm{~dB}$ per node. In semiconductors like GaAs, the loss per node should not exceed 1 to $2 \mathrm{~dB}$.

\section{Total Optical Insertion Loss of IOM}

The total optical insertion loss of an IOM will depend on the material system, the number of nodes, and the number of fiber interconnects. Using the previous estimates for the various loss factors, an expression for total insertion loss is

$$
\begin{aligned}
\text { TIL = } & \text { \# of fiber } \\
& \text { connections*loss/fiber } \\
& \text { connection }+ \text { \# of } \\
& \text { switching nodes }
\end{aligned}
$$

Using this equation, Table A-1 is generated.

Based on these numbers, it is clear that the architecture that uses an SOA after the 


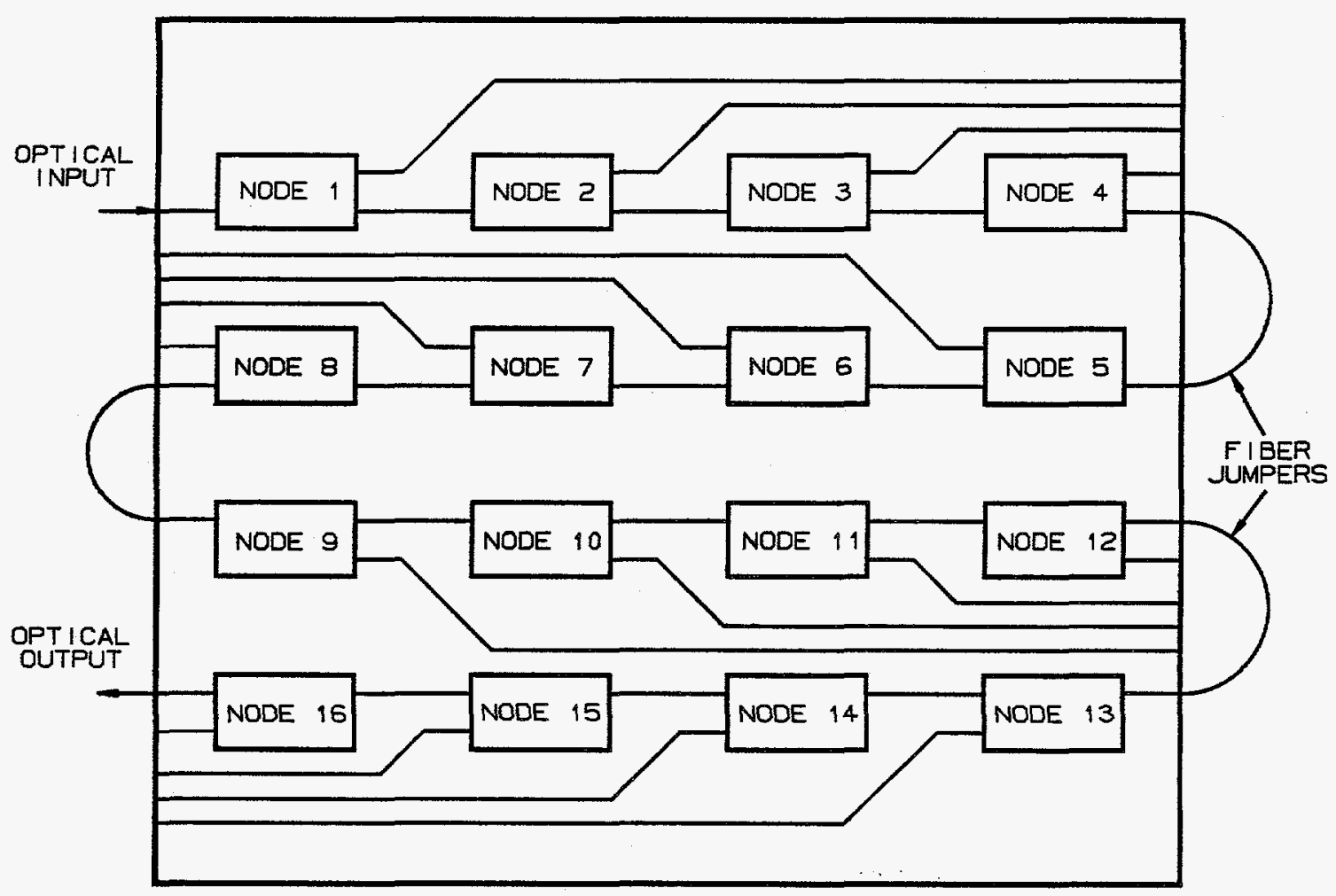

Figure A-5. Sixteen-Node Integrated Optical Maze Schematic

$10 M$ will be required for a 16 -node $1 O M$ and possibly for a four-node IOM as well.

\section{Switching Voltage and Current}

As discussed earlier, the switching voltage and current are dependent on the material system, the device size, and the switching mechanism. For example, in the glass system (thermo-optic switch) the important attribute is the temperature increase that can be generated in a thin-film heater that locally alters the waveguide's parameters. The combination of voltage and current that will result in a given dissipation is determined by the resistance of the thinfilm heater. However, real power must be dissipated in order to switch the light. In the $\mathrm{LiNbO}_{3}$ or $\mathrm{LiTaO}_{3}$ material system, very little power needs to be dissipated in order to switch the device. This is because the switching mechanism is the electro-optic effect which in the ideal case requires no real power to modify the state of the switch.

Table A-1. Insertion Loss of IOM for Different Material Systems

\begin{tabular}{||l|c|c||}
\hline Material System & $\begin{array}{c}4 \text { Nodes } \\
\text { (Figure A-1) }\end{array}$ & $\begin{array}{c}16 \text { Nodes } \\
\text { (Figure A-5) }\end{array}$ \\
\hline Glass & $3 \mathrm{~dB}$ & $12 \mathrm{~dB}$ \\
\hline $\mathrm{LiNbO}_{3} / \mathrm{LiTaO}_{3}$ & $6 \mathrm{~dB}$ & $24 \mathrm{~dB}$ \\
\hline GaAs & $9 \mathrm{~dB}$ & $36 \mathrm{~dB}$ \\
\hline
\end{tabular}


Typical voltage length products are less than $15 \mathrm{~V}-\mathrm{mm}$. This means that a device that has a $5-\mathrm{mm}$ long electrode would switch with just $3 \mathrm{~V}$. If a $10-\mathrm{k} \Omega$ resistor was used in parallel with the switch electrodes, then only $0.9 \mathrm{~mW}$ would be required to switch the device. Alternatively, if no resistor was used, then only $0.135 \mu \mathrm{W}$ of reactive power would be required to switch the device in $1 \mathrm{~ms}$.

Estimating the dissipation of a semiconductor switch is more complex because first the type of device and switching mechanism must be identified. In semiconductor modulators, the switching mechanism can either be electro-optic (Walker, Appl. Phys., Lett. 54, 1613, 1989) or electrorefractive (Zucker, et. al., Appl. Phys., Lett. 55, 2280, 1989). In both cases the switching speed is rapid. The electrooptic-based semiconductor switches operate in a fashion similar to their ferroelectric counterparts. The electrorefractive-based switches operate through an exitonic electrorefractive effect in reverse-biased quantum well heterostructures. Although these can be quite efficient, they must be operated at wavelengths near the semiconductor band edge.

Voltage-length products as low as $8 \mathrm{~V}$-mm have been reported for this type of switch. Because the quantum well is reverse biased, the only current flow is that caused by junction leakage, which is only $1 \mu \mathrm{A}$ at junction breakdown. Thus, the switching power required is quite low, $18 \mu \mathrm{W}$. Semiconductor devices based on the electro-optic effect are not nearly as efficient as their electrorefraction counterparts but provide results comparable to ferroelectric modulators.

The extinction ratio (on/off ratio or crosstalk) achievable with the electro-optic switch based on $\mathrm{LiNbO}_{3}$ is typically better than $-20 \mathrm{~dB}$. The semiconductor devices typically do not do as well, with the electrorefractive devices being the worst.

\section{Physical Size and Weight}

The physical size and weight of the ferroelectric and electro-optic semiconductor modulators should be about the same. The size and weight of the semiconductor electrorefractive modulators could be two to three times smaller. The absolute size will depend on the material choice, number of switching nodes, etc., and will be discussed further in the IOM design section of this appendix.

\section{Optical Source Characteristics}

The semiconductor laser source must be capable of putting out power sufficient that after the IOM loss and the SOA gain are accounted for, $650 \mathrm{~mW}$ is output to the next stage of the system. Because the pulse width and rise and fall times are relatively long compared to diode laser dynamics, the modulation speed of the source is not an issue. As described in an earlier section, the maturity of high-power $0.985 \mu \mathrm{m}$ diode lasers that provide diffraction limited spots, coupled with the resistance of ferroelectrics to optical damage as the wavelength is increased, makes them a good candidate. Because they are used as DPSS laser pump sources, their price can be expected to fall as their dual-use (commercial and this application) volumes increase and their reliability can be expected to improve as well.

\section{IOM Material System}

The material system to be used for the IOM should be determined by a variety of criteria, including performance, adherence to the desired specifications, and perhaps just as importantly, availability. Guidedwave switching devices based on $\mathrm{LiNbO}_{3}$ have been commercially available since 1987. Today, they can be purchased from four to six different vendors, with two or three being capable of undertaking custom device development. The major drawback 
of this material system is the optical performance at the high peak powers required, i.e., optical damage. Providing that this issue does not pose problems, a $\mathrm{LiNbO}_{3}$-based IOM is an excellent candidate. Less developed, but certainly commercially demonstrated, are devices based on $\mathrm{LiTaO}_{3}$. These have been used in situations where higher optical power handling capabilities were needed. $\mathrm{LiTaO}_{3}$ is known to be much more resistant to optical damage than is $\mathrm{LiNbO}_{3}$.

Devices based on the electrorefractive effect in semiconductor quantum wells are not mature and are not commercially available. Their main advantage appears to be their size. Although they should be able to handle high optical powers, this property has not been scrutinized to the same level of detail as have the ferroelectrics.

Semiconductor devices based on the electro-optic effect have been commercially available since 1991 . However, this primarily means that data sheets are available and at least small quantities have been delivered. In general, the performances of the devices are not particularly impressive. Their insertion loss is dramatically higher than comparable $\mathrm{LiNbO}_{3}$ devices, and other pertinent parameters are about the same as those found in their $\mathrm{LiNbO}_{3}$ counterparts. Even having made this statement, the best designed $\mathrm{LiNbO}_{3}$ devices reported have outperformed the semiconductor devices across the board. This is particularly true in the highbandwidth regime where velocity-matching techniques on $\mathrm{LiNbO}_{3}$ have allowed very low-voltage, high-bandwidth devices to be developed.

The closeness of the optical and microwave velocities on semiconductors has been their strong point because of the wide bandwidth that this allows. Clever techniques on $\mathrm{LiNbO}_{3}$ have accomplished the same result, severely diminishing the importance of the electro-optic-based semiconductor devices. In addition, the high-speed aspect of the device has no relevance to the IOM application.

The ability to fabricate electronics on the same substrate as the electro-optic device has been touted as a great advantage for semiconductor-based optical devices. However, to date the incompatibilities of the different materials required for optical versus electronic devices have made progress slow. The real value of this capability has yet to be adequately demonstrated. Persuasive counter arguments have been made for integrating the electronics and the optics in a hybrid fashion using multichip modules. When this is done, the individual components can be individually optimized. This may mean high-speed GaAs electronics and highspeed $\mathrm{LiNbO}_{3}$ switches, for example.

Based on the preceding arguments, it seems apparent that if the ferroelectric material system is able to accommodate the optical powers required, then it could be the material system of choice for the IOM application. The susceptibility of $\mathrm{LiNbO}_{3}$ and $\mathrm{LiTaO}_{3}$ integrated optical switches to optical damage is analyzed and discussed in the next section.

\section{Optical Damage and Ferroelectric Waveguide Devices}

The existence of light-induced optical refractive index inhomogeneities in $\mathrm{LiNbO}_{3}$ have been known since 1966. This effect was exploited during the 1970s for holographic storage. The dominant mechanism is the photo-induced reaction

$\mathrm{Fe}^{2+}+\mathrm{hv}^{2} \rightarrow \mathrm{Fe}^{3+}+\mathrm{e}^{-}$

where $\mathrm{Fe}^{2+}$ is the residual iron impurity in the reduced state and $\mathrm{Fe}^{3+}$ is the iron impurity in the oxidized state. The liberated electron drifts out of the illuminated region where it is retrapped, thus establishing an internal electric field which causes 
refractive index inhomogeneities through the electro-optic effect of the crystal. This process has been studied extensively for bulk $\mathrm{LiNbO}_{3}$ and $\mathrm{LiTaO}_{3}$ in the past. More recently, it has been investigated in waveguide devices formed through $\mathrm{Ti}$ indiffusion or proton exchange in $\mathrm{LiNbO}_{3}$ and $\mathrm{LiTaO}_{3}$. The effect is known to be enhanced at shorter wavelengths (UV to near infrared) and in the presence of externally applied electric fields. Reports of power handling capabilities are displayed in Table A-2.

It is believed that pulsed inputs of much higher magnitude should be possible, providing that the pulse duration is short compared to the photorefractive dynamics.

An investigation into the large difference between the power handling capability of $\mathrm{Ti}-\mathrm{LiNbO}_{3}$ and $\mathrm{PE}-\mathrm{LiNbO}_{3}$ has indicated that the indiffusion of $\mathrm{Ti}$ tends to reduce the iron impurity, increasing the concentration of photoionizable $\mathrm{Fe}^{2}+$ (Young, et. al., Opt. Lett. 16, 995, 1991), whereas the proton exchange process tends to oxidize the iron impurity, reducing the concentration of photoionizable $\mathrm{Fe}^{2}+$ (Olivares, et. al., Appl. Phys., Lett. 61, 624, 1992). An expression for the space charge field photorefractive dynamics of $\mathrm{LiNbO}_{3}$ guided-wave devices is given by the following expression (Becker, et. al., Appl. Phys., Lett. 47, 1024, 1985):

$$
\begin{aligned}
\operatorname{Esc}(t) & =\left[\sigma_{p h} / \sigma * E_{a p p}+k^{\prime} \alpha J_{0} / \sigma\right. \\
& \left.-E_{s c}(0)\right]^{*}[1-e-(\sigma t / \varepsilon \varepsilon 0)]+E_{s c}(0),
\end{aligned}
$$

where

$\sigma_{\mathrm{ph}}$ is the photoconductive constant,

$\sigma$ is the total conductivity,

$K^{\prime}$ is the modified glass constant,

$\alpha$ is the absorption coefficient caused by the $\mathrm{Fe}^{2}+$ ions,

Esc(0) is the space charge field present at time $t=0$, and

$I_{0}$ is the optical input intensity (assumed to be $\mathrm{CW}$ ).

Of primary interest here is the magnitude of the exponential term

$$
\left[1-e^{-(\sigma t / \varepsilon \varepsilon o)]}\right. \text {, }
$$

or actually, the magnitude of $\tau=\varepsilon \varepsilon \sigma / \sigma$, the photorefractive time constant. The total conductivity $\sigma$ is equal to the sum of the photoconductivity, $\sigma_{\mathrm{ph}}$, and the dark conductivity, $\sigma_{d}$. The dark conductivity for annealed $\mathrm{PE}-\mathrm{LiNbO}_{3}$ has been reported (Fujiwara, et. al., Opt. Lett. 18, 346, 1993) to be

$$
\sigma_{d}=0.6 * 10^{-14}(\Omega-\mathrm{cm})^{-1}
$$

Table A-2. Power Handling Capabilities

\begin{tabular}{|r|c|c|c||}
\hline Optical Power & Wavelength & Material & Source \\
\hline $2 \mathrm{~mW} \mathrm{CW}$ & $0.810 \mu \mathrm{m}$ & Ti-LN & UTP \\
\hline $50 \mathrm{~mW} \mathrm{CW}$ & $0.810 \mu \mathrm{m}$ & PE-LN & LLNL \\
\hline $100 \mathrm{~mW} \mathrm{CW}$ & $0.800 \mu \mathrm{m}$ & PE-LT & UTP \\
\hline $200 \mathrm{~mW} \mathrm{CW}$ & $1.3 \mu \mathrm{m}$ & PE-LN & UTP \\
\hline
\end{tabular}

Note that these are all $\mathrm{CW}$ measurements. 
Next, the photoconductive constant needs to be calculated as a function of input intensity. Again from Fujiwara, the photoconductive constant for $\lambda=0.488 \mu \mathrm{m}$ illumination can be expressed as

$$
\sigma_{\mathrm{ph}}=\sigma_{\mathrm{d}} * 6.1 * 10^{-3} * \mathrm{I}_{\mathrm{o}}(\Omega-\mathrm{cm})^{-1} .
$$

The dependence of the photorefractive effect on wavelength was also measured by Fujiwara, et. al. (Appl. Phys., Lett. 54, 975,1989 ). It was found that the effect would be roughly four orders of magnitude smaller at a longer wavelength such as $0.985 \mu \mathrm{m}$. Thus, the expression for $\sigma_{\mathrm{ph}}$ above can be adjusted for this. Making this adjustment and substituting for $\sigma_{d}$, we get

$$
\sigma_{\mathrm{ph}}=3.66 * 10-21 * I_{0}\left(W / \mathrm{cm}^{2}\right)\left(\Omega-\mathrm{cm}^{-1} .\right.
$$

In Figure A-6, the time constant, $\tau$, is plotted versus optical power in the IOM. Also shown is the time constant calculated for $\mathrm{Ti}^{-\mathrm{LiNbO}_{3}}$ (also from Fujiwara). The $\mathrm{Ti}$-LiNbO${ }_{3}$ time constant is multiplied by 10 so it is easier to read on the graph. As expected, the time constant for the PE$\mathrm{LiNbO}_{3}$ is much longer than the time constant for the $\mathrm{Ti}-\mathrm{LiNbO}$. From Figure A-6, it is seen that even $\mathrm{Ti}^{\mathrm{L}} \mathrm{LiNbO} \mathrm{N}_{3}$ may be acceptable for a 10-ms wide optical pulse at $0.985-\mu \mathrm{m}$ wavelength of peak power greater than $2 \mathrm{~W}$. However, given the excellent availability of PE-LiNbO ${ }_{3}$ devices and their additional robustness with respect to high optical power, they would be the material of choice for the IOM application. From Figure $A-6$ and the equation for $\operatorname{Esc}(t)$, it is seen that even with a $2-W$ pulse, the space charge field would only build up to 0.00013 of its saturated value during the 10-ms pulse duration. This is not sufficient to adversely affect the operation of the switch. Because PE- $\mathrm{LiNbO}_{3}$ appears to be sufficient, there is no need to investigate $\mathrm{PE}-\mathrm{LiTaO}$. Although it is likely to be even more robust, devices based on it are not as easily obtainable.
A final damage mechanism that needs to be considered independently from the material system chosen is the possibility of catastrophic endface damage from the high intensity pulse. The cross-sectional area of a $0.985-\mu \mathrm{m}$ waveguide is approximately $25{ }^{*} 10^{-8} \mathrm{~cm}^{2}$. For a $2-W$ input, this represents an intensity of $8 \mathrm{MW} / \mathrm{cm}^{2}$. This is sufficiently lower than the damage threshold for $\mathrm{LiNbO}_{3}\left(100\right.$ 's of $\left.\mathrm{MW} / \mathrm{cm}^{2}\right)$ that it need not be of concern. In addition, in a 10-ms pulse, only $20 \mathrm{~mJ} / \mathrm{cm}^{2}$ of energy density is present.

Thus, it appears that an IOM based on $\mathrm{PE}-\mathrm{LiNbO}_{3}$ is reasonable and will be used in the IOM design exercise.

\section{Integrated Optical Maze Design}

In this section, a design of an $1 O M$ based on PE-LiNbO ${ }_{3}$ is developed. A $0.985-\mu \mathrm{m}$ operating wavelength is assumed because of the availability of high-power diffraction limited diode lasers and optical power amplifiers at this wavelength. The design will focus on the architecture shown in Figure A-1. The switching nodes will comprise $2 \times 2$ Mach-Zehnder switches as shown in Figure A-3. The choice of the $2 \times 2$ Mach-Zehnder switch was made because it is easily fabricated on temperature-stable $\mathrm{X}$-cut $\mathrm{LiNbO}_{3}$. A design will first be developed for a four-node IOM and then expanded to a 16-node IOM for comparison. In the four-node case, the $10 M$ will be arranged so that without any programming voltages applied, two high control voltages and two low control voltages will be required to achieve a Red condition.

The first step in developing such a design is the design of the switching node. Shown in Figure A-7 is a detailed drawing of a $2 \times 2$ Mach-Zehnder switch suitable for use at $0.985 \mu \mathrm{m}$. This switching node has two electrodes, one for the programming voltage and one for the control voltage. 


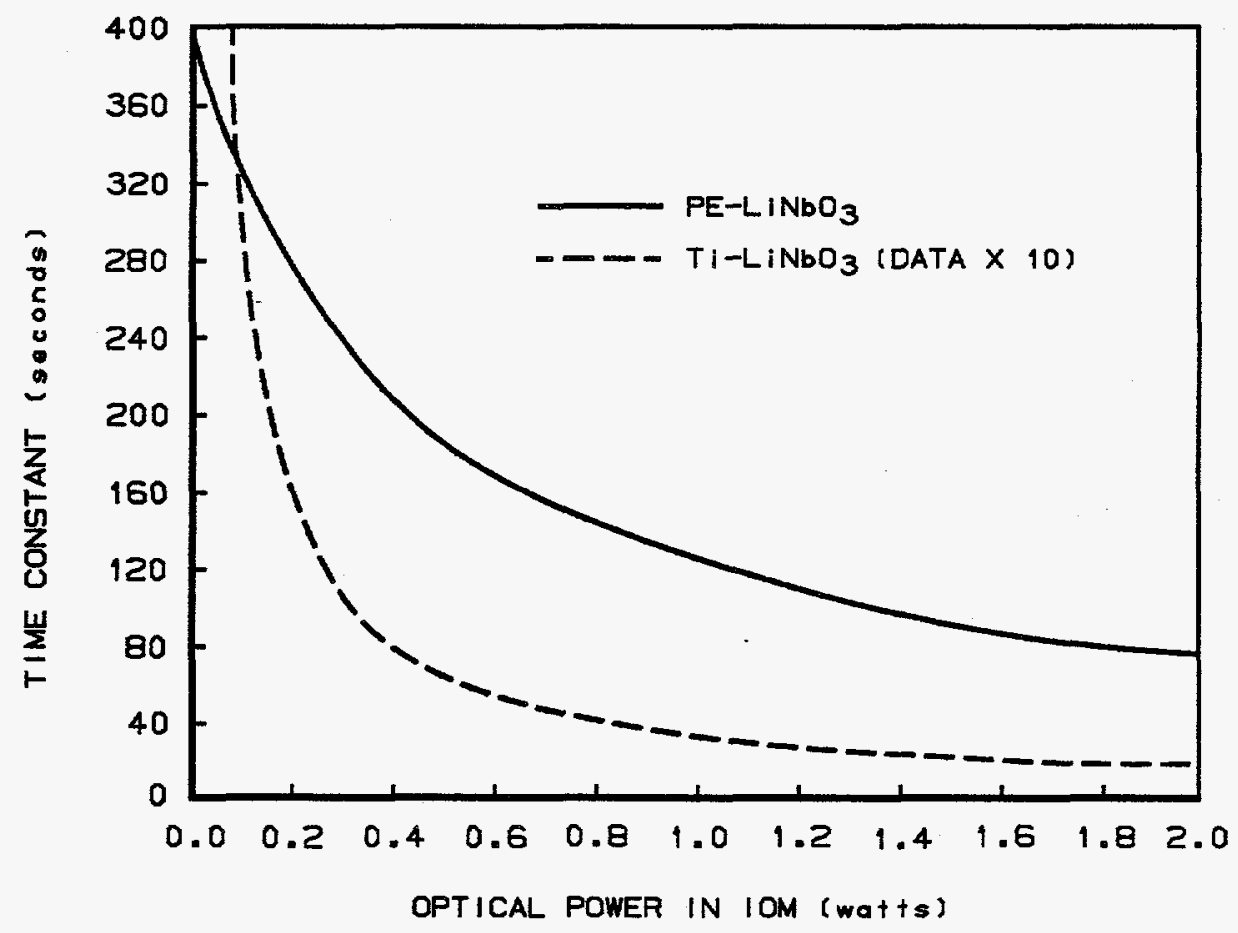

Figure A-6. Photorefractive Time Constant Versus Optical Power in IOM

The voltage-length product for this device will be approximately $15 \mathrm{~V}$-mm; the lengths of the programming and control electrodes can be apportioned as desired. For example, they could be made equal, in which case the programming voltage and the control voltage would be equal to about $6 \mathrm{~V}$ each. Low-loss raised sine bends are used in the waveguide design. If a $0.5 \mathrm{~dB} / \mathrm{cm}$ propagation loss and a $0.05-\mathrm{dB}$ loss/bend is assumed, then the total waveguide loss (not counting input/output coupling) would be $1.175 \mathrm{~dB} /$ node. The electrodes are sufficiently spaced so that no metal-loading loss should be present. Nodes 2 and 3 of the IOM look like the switch depicted in Figure A-7. Nodes 1 and 4 have to be modified so that the spacing of the inputoutput waveguides is increased to $250 \mu \mathrm{m}$ for ease of fiber attachment. An input/output stem, $5000 \mu \mathrm{m}$ long is also used to provide sufficient mode filtering at the input/output to the $1 O M$.

The four-node IOM is shown in Figure A-8. Only the dimensions that are different from those shown in Figure A-7 are included.
Note that this figure is not drawn to scale. In reality, the IOM is 64 times longer than it is wide. This large aspect ratio difference allows four such $10 \mathrm{Ms}$ to be assembled on the same substrate as depicted in Figure A-5. Thus, on a $\mathrm{LiNbO}_{3}$ substrate $65 \mathrm{~mm}$ long and $5 \mathrm{~mm}$ wide, a 16-node IOM is easily achieved. Without any programming voltages applied, nodes 1 and 4 would have to be in the Low state and nodes 2 and 3 in the High state in order for a Red condition to occur. As discussed earlier, by using programming voltages, the node states for a Red condition to occur could be continually changed.

The optical insertion loss of a four-node IOM is now calculated. Earlier, the loss/node was calculated to be $1.175 \mathrm{~dB} /$ node. Including four nodes and input/output coupling loss, a total fiber-tofiber insertion loss of $6.7 \mathrm{~dB}$ is obtained. This is quite close to the value estimated in Table A-1. If we assume the use of a 10-dB optical power amplifier at the IOM output, then the input laser needs to be able to deliver $304 \mathrm{~mW}$ to the IOM. The 


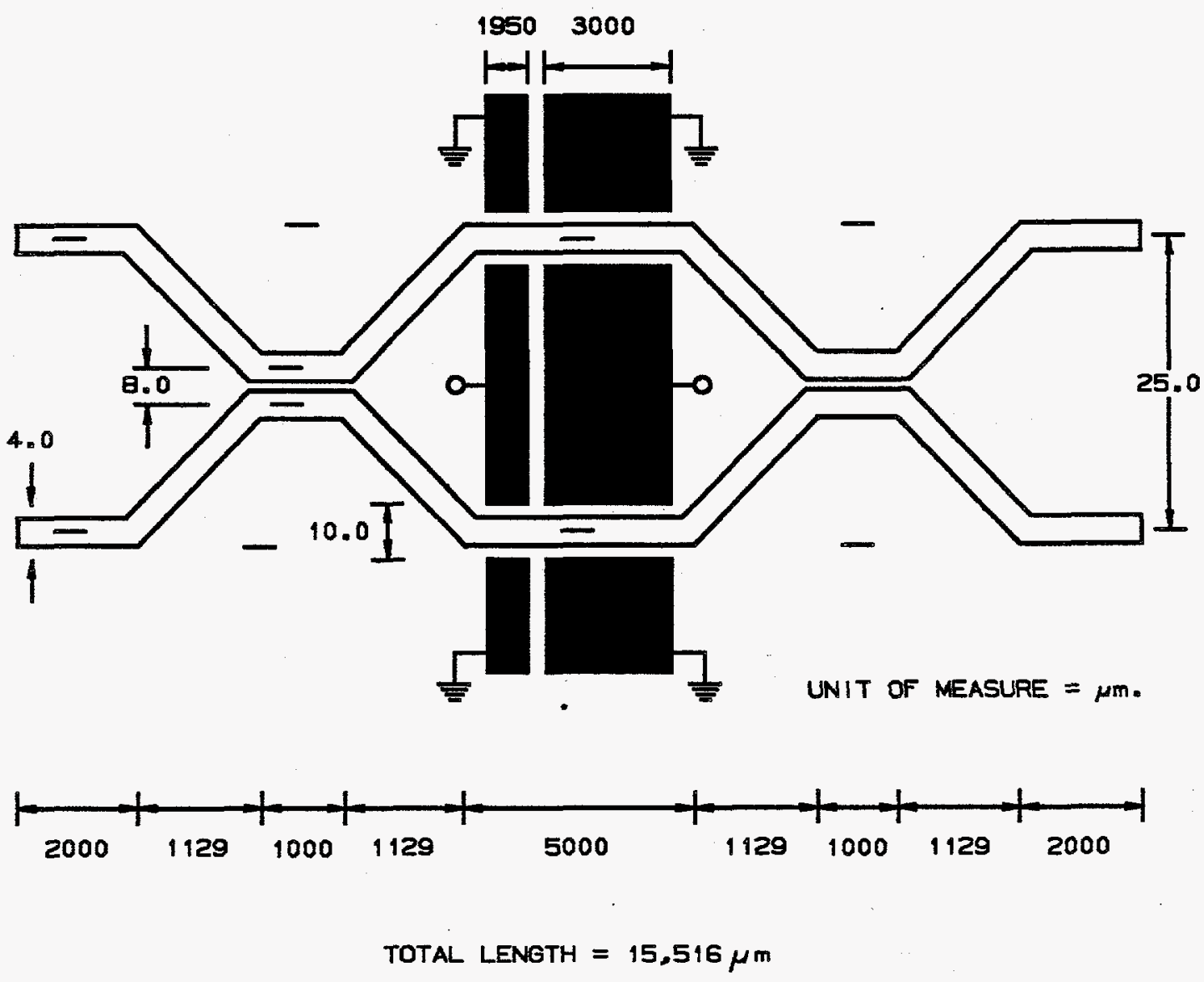

Figure A-7. Design of IOM Switch Node

optical insertion loss of a 16-node IOM is four times that of a four-node $10 \mathrm{M}$, or $26.8 \mathrm{~dB}$. In this case, additional gain is going to be required. For example, if a 1-W source is used, then an SOA with a gain of $25 \mathrm{~dB}$ will be required at the $1 \mathrm{OM}$ output. Alternatively, a 304-mW source could be used and a 7.5-dB gain SOA could be installed after each of the fournode chains. Depending on the cost and power budget, the number of SOAs and their respective gains could be adjusted to obtain the desired $650-\mathrm{mW}$ output pulse.

The optical source would need to be connected to the IOM using polarizationmaintaining single-mode optical fiber (PMSM). In addition, any fiber jumpers used (as seen in Figure A-5) and the connection to the SOA would require its use as well. The final output from the last SOA could be multimode fiber.
The final size of the IOM would be determined by the packaging approach used. A typical package would be $85 \mathrm{~mm}$ $\times 15 \mathrm{~mm} \times 10 \mathrm{~mm}$ for the four-node $10 \mathrm{M}$ and $105 \mathrm{~mm} \times 15 \mathrm{~mm} \times 15 \mathrm{~mm}$ for the 16 node IOM. The weight would be determined by the packaging material used. A metallic-packaged device would weigh in the vicinity of 1 ounce.

Table A-3 shows the performance specifications for both a four-node and a 16-node IOM based on the design presented here.

Up to this point, electronic control voltages were assumed. However, the control voltages could be delivered optically to the IOM through the use of photocells integrated on or in the IOM package. This would enable an IOM without electrical connections to be realized. 


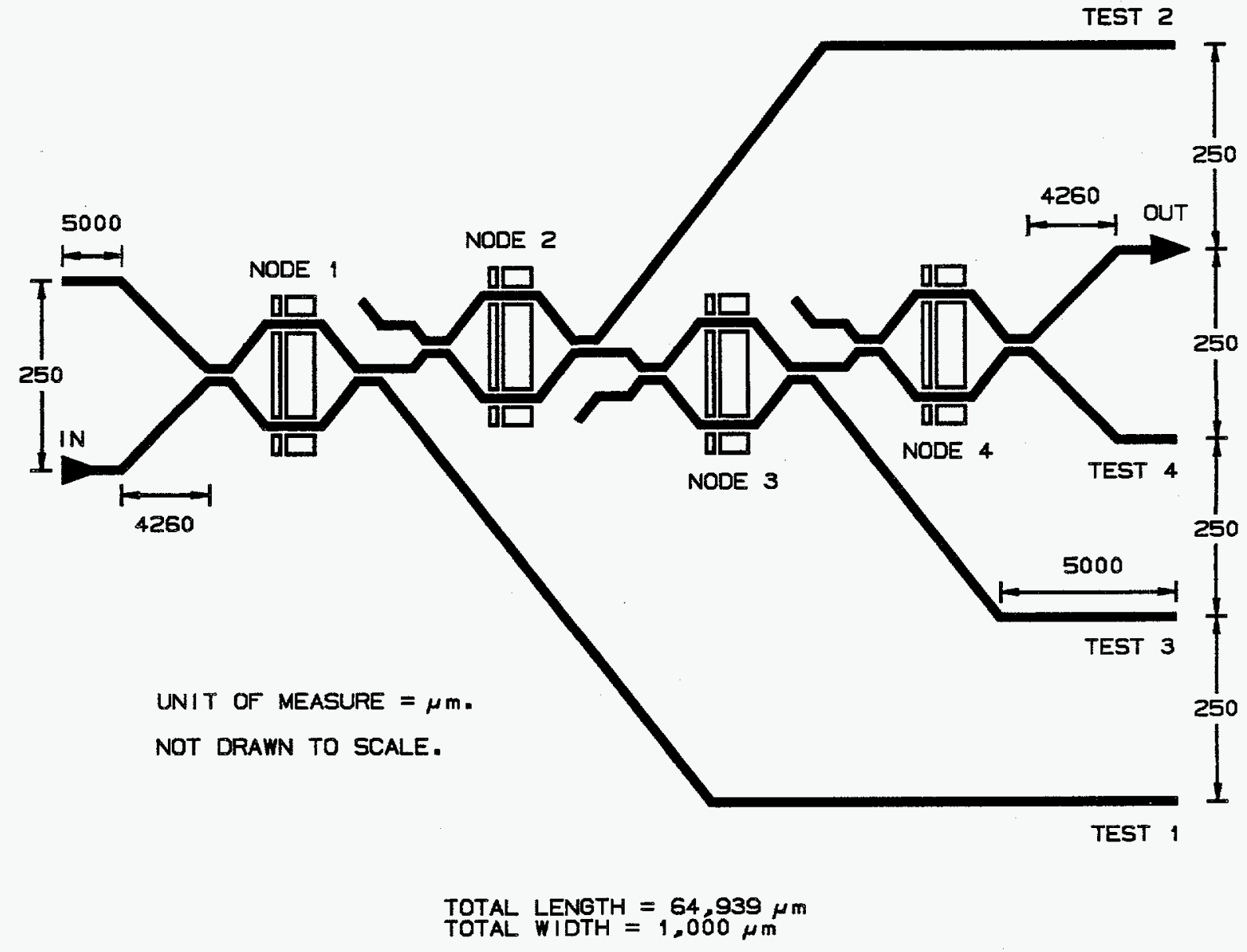

Figure A-8. Four-Node Integrated Optical Maze Design

\section{Summary and Conclusions}

In this design review, the concept of an integrated optical maze was discussed. The specifications were evaluated, and several different approaches were considered. It appears from this level of investigation that an IOM is certainly achievable using existing $\mathrm{PE}-\mathrm{LiNbO} 3$ switch array technology and existing highpower diode laser technology at the $0.985-\mu \mathrm{m}$ wavelength. The use of the IOM approach may provide improvements to overall system performance. 
Table A-3. IOM Design Specifications

\begin{tabular}{||l|c|c||}
\hline \multicolumn{1}{|c|}{ Parameters } & 4-Node & $16-$ Node \\
\hline Optical Power Out & $650 \mathrm{~mW}$ & $650 \mathrm{~mW}$ \\
\hline Optical Pulse Length & $10 \mathrm{~ms}$ & $10 \mathrm{~ms}$ \\
\hline Optical Rise/Fall Time & $1 \mathrm{~ms}$ & $1 \mathrm{~ms}$ \\
\hline Optical Wavelength & $0.985 \mu \mathrm{m}$ & $0.985 \mu \mathrm{m}$ \\
\hline Number of Switches & 4 & 16 \\
\hline Fiber Coupling Loss & $2 \mathrm{~dB}$ & $8 \mathrm{~dB}$ \\
\hline Loss per Node & $1.157 \mathrm{~dB}$ & $1.157 \mathrm{~dB}$ \\
\hline Coupling Loss to SOA & $1 \mathrm{~dB}$ & $4 \mathrm{~dB}$ \\
\hline IOM Insertion Loss & $6.7 \mathrm{~dB}$ & $26.8 \mathrm{~dB}$ \\
\hline Switching Voltage (Power) & $6 \mathrm{~V}(0.3 \mu \mathrm{W})$ & $6 \mathrm{~V}(1.2 \mu \mathrm{W})$ \\
\hline Size of Switch Array & $85 \times 15 \times 10(\mathrm{~mm})$ & $105 \times 15 \times 15(\mathrm{~mm})$ \\
\hline Material & $\mathrm{PE}-\mathrm{LiNbO}$ & $\mathrm{PE}$-LiNbO 3 \\
\hline Number of SOAs (Gain) & $1(10 \mathrm{~dB})$ & $4(7.5 \mathrm{~dB})$ \\
\hline Source Power & $304 \mathrm{~mW}$ & $304 \mathrm{~mW}$ \\
\hline \hline
\end{tabular}

